\title{
Modern pollen rain in heathlands and adjacent forest phytocoenoses in Northern and Central Poland
}

\author{
*Andrzej Nienartowicz, *Anna Filbrandt-Czaja, *Agnieszka Piernik, \\ *Piotr Jabłoński, **Mieczysław Kunz, *Miłosz Deptuła
}

\author{
Nicolaus Copernicus University, Faculty of Biology and Earth Sciences, \\ *Institute of Ecology and Environment Protection, Laboratory of Ecological Processes Modelling, \\ e-mail: afczaja@umk.pl \\ **Institute of Geography, Department of Cartography, Remote Sensing and GIS, \\ Gagarina 9, 87-100 Toruń, Poland
}

\begin{abstract}
Differences between modern pollen rains in three types of phytocoenoses were studied in two regions, i.e. Tuchola Forest and Torun Basin. The phytocoenoses in question were heaths, young pine-birch growths and mature pine tree stands, which form a temporal sequence and spatial mosaic in the landscape. In order to compare the structure of pollen samples, phytocoenoses and the landscape, the methods of numerical taxonomy were applied. An increase in the average number of sporomorphs was recorded, as well as in the taxonomic diversity of the pollen spectrum in this temporal sequence arranged from non-forest communities through young growths to mature forests. In the subsequent developmental stages, also the percentage contribution of Pinus pollen increased, as well as the total contribution of arboreal pollen (AP). The increase in the percentage contribution of arboreal pollen is accompanied by the decrease in the contribution of non-arboreal pollen (NAP), including Calluna and Ericaceae. Also a small increase in the number of species was observed. With the increased pine domination, however, the diversity of pollen types (expressed by rarefaction and Shannon-Wiener indices) decreases. As a result of the performed analyses, it was also found that the species composition of phytocoenoses and a pollen spectrum differs considerably, because the import of pollen from further areas exerts a significant influence on the structure of a palynological sample. The genus Calluna is the only type of plants characterized by a strong relationship between the occurrence in a phytocoenosis and in a surface pollen sample. Similarity between the taxa composition of a pollen spectrum and the landscape structure, consisting of a mosaic of phytocoenoses, seems to be higher than between a pollen sample and a phytocoenosis from which that sample was collected. The latter thesis, however, needs to be confirmed by further researches.
\end{abstract}

Key words: Tuchola Forest, Toruń Basin, dry heathlands, young forests, mature forests, pollen spectra, pollen representation, PAR, rarefaction, numerical classification, ordination.

\section{Introduction}

In recent years, the interest in researches on the structure of modern pollen rain has increased. Two aspects dominate in these researches. These are assessments of the relationship between the vegetation structure and the pollen spectrum, as well as the influence of climate exerted on the structure of modern pollen rain. The research conducted from the first aspect concerns mainly the variability of the pollen spectrum in landscapes of different openness, estimation of the area size, the vegetation of which is represented by a pollen sample and the variability of the pollen spectrum in vegetation zones occurring along gradients of ecological factors, such as substrate moisture or salinity. The research dealing with the influence of climate on the pollen rain includes assessments of the pollen production by the same tree species at different latitudes, as well as changes in the 
pollen spectrum along the altitude gradient in mountain areas. Numerous references on the aforementioned issues are provided in papers by Jackson (1991), Fontana (2003, 2005), Hrynowiecka-Czmielewska et al. (2007), Bunting and Hjelle (2010), Pidek et al. (2010). In these papers, also results of the researches on the modern pollen rain are presented, which were carried out from different aspects and in different landscape types in several regions of the world.

In the landscape dominated by forests, the relationship vegetation - modern pollen rain was particularly often studied in North America (e.g. Davis 1963; Davis 1964; Davis \& Webb 1975; Bradshaw \& Webb 1985; Jackson 1990; Calcote 1995; Janssen 1996) and in Europe (Brem \& Sobolewska 1939; Tauber 1967; Andersen 1970; Dąbrowski 1975; Parsons et al. 1980; Bradshaw 1981; Prentice et al. 1987; Bunting 2002). In Poland, apart from Białowieża National Park, this kind of research has been carried out also in Tuchola Forest. In this region, Noryśkiewicz (1997) investigated the modern pollen rain on the litter in the reserve „Cisy Staropolskie” (Old Polish Yew Trees) - one of the oldest protected areas in Europe, where protection of yew forest started already in 1828. Palynological studies conducted in Tuchola Forest by Hrynowiecka-Czmielewska et al. (2007) had a broader scope, as they analysed modern pollen spectra in phytocoenoses of pine and deciduous forests, wet and dry meadows, as well as heaths. There were only two heath phytocoenoses among the ecological systems studied by these authors. Since the contribution of heaths in the landscape of the present-day Tuchola Forest is relatively high, and in the past their significance was even higher, the decision was made to study the modern pollen rain of heaths with a larger number of samples.

The present paper also aimed at comparing the relationship between vegetation and modern pollen rain with the vegetation structure and the pollen spectrum of young growths and mature pine forests, which nowadays dominate in Tuchola Forest. Modern pollen spectra of this region were compared with pollen samples collected in phytocoenoses of the same type in the region where at present large heathlands occur. The object of the comparisons was the Torun Basin, where large areas of heaths occur on military training grounds.

Pollen rain analyses presented in this paper have been carried out with the conviction that the estimated differences between the pollen rain structure in heaths and forests of different age in the modern landscape can contribute to evaluation of the influence exerted by the former human economic activity on the vegetation structure in the historic landscape, based on the analysis of fossil materials.

\section{The study area}

The researches were carried out in two regions: in the Tuchola Forest (A) in North-Western Poland and in the Torun Basin (B) in the central part of Poland (Fig. 1). In both units, pine forests are the dominant ecosystem type forming extensive forest complexes. In the former region, they mainly cover soils developed from sand of an outwash plain, in the latter region - inland dunes. After cutting down the forest, dry heaths occur in both areas, classified within two associations from the class Nardo-Callunetea (Kujawa-Pawlaczyk 2004; Chojnacka et al. 2010a, b). Those are heaths with Pohlia nutans, i.e. Pohlio-Callunetum and bearberry heaths Arctostaphylo-Callunetum. They are accompanied by psammophilous grasslands, classified mainly as the associations Spergulo-Corynephoretum and Calamagrostietum epigeji according to the classification of plant communities in Poland by Matuszkiewicz (2007).

The area of the whole Tuchola Forest region, within the limits designated by Trampler et al. (1990) and within the scope of the study ,Nature and forest regionalization of Poland", covers over $5,000 \mathrm{~km}^{2}$. The forestation rate of this region amounts to over $50 \%$. And thus, the Tuchola Forest is one of the largest forest areas in Poland. In the past, heaths were quite important here. Today, they cover small areas within firebreaks, demarcation lines between particular forest sections, as well as take-off airstrips, which until recently were used by forest-fertilization and firefighting planes.

The population density in the area is low. There are a few, rather small, towns here - Chojnice, Czersk, Tuchola and Brusy. The whole Tuchola Forest region constitutes the Biosphere Reserve and the Natura 2000 Site, designated on the basis of the EU Birds Directive. Furthermore, there are several Natura 2000 areas designated based on the Habitat Directive, as well as over twenty approved nature reserves and several designed ones. There are also four landscape parks and one Tuchola Forest National Park. Most of the forests are the property of the State Treasury and are under the administration of 20 forest inspectorates subordinate to three Regional Directorates of State Forests in Gdańsk, Szczecinek and Torun. The material for studies was collected along the peripheries of the Tuchola Forest National Park, as well as Zaborski and Tuchola Landscape Parks. The material was collected from forests included within the forest inspectorates of Przymuszewo and Tuchola.

Figure 1 presents the location of the Tuchola Forest region, which in the system of Trampler et al. (1990) is denoted with symbol III.1.

In the Torun Basin, defined in the system of Trampler et al. (1990) as region III.3, the research was carried out in its western part, i.e. in the forest complex called Bydgoszcz Forest (Fig. 1). The Vistula River Valley constitutes the 

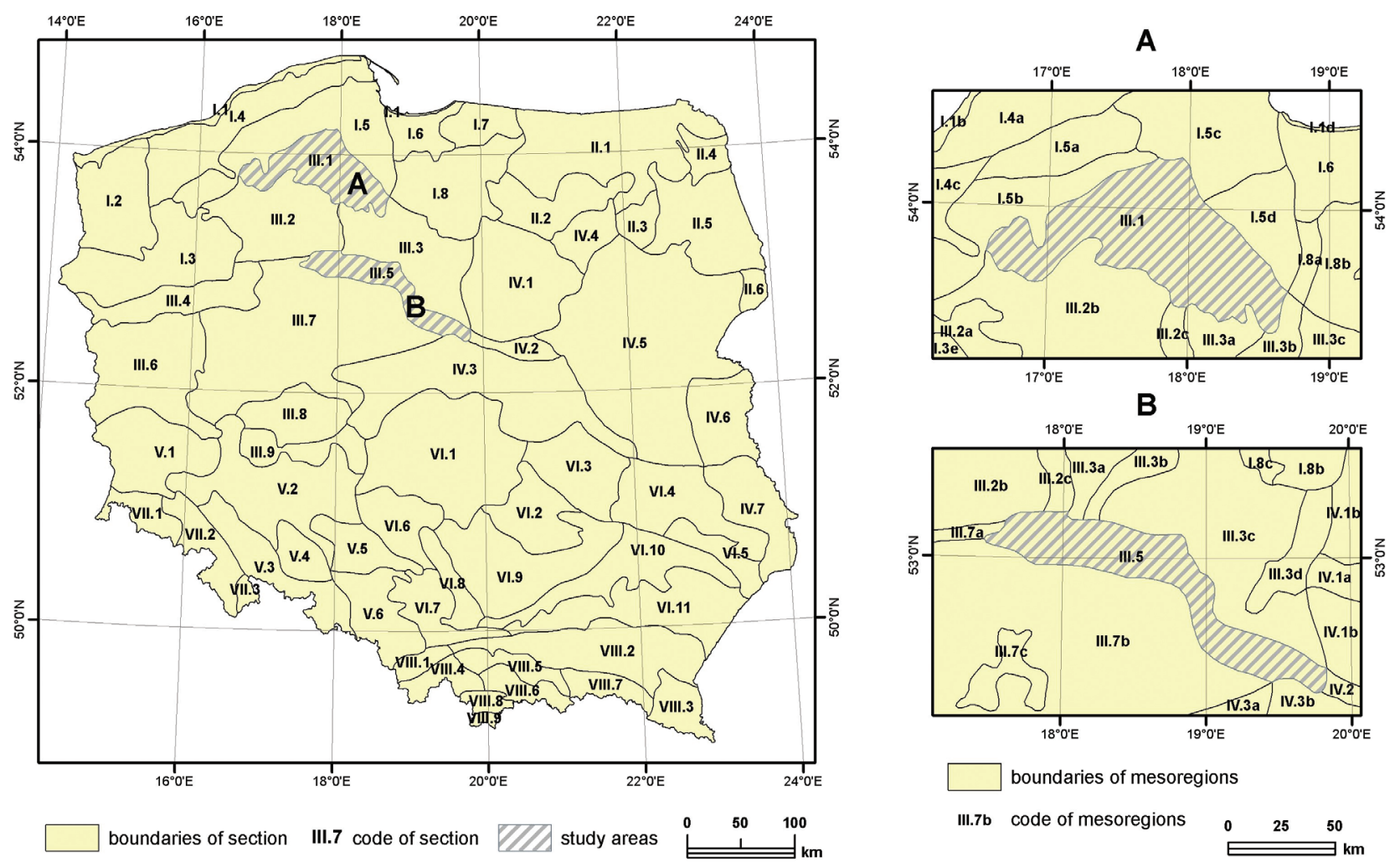

Figure 1. The location map of the study area in nature-forest regions after Trampler et al. (1990); a - Tuchola Forest, B - Torun Basin

axis of the Torun Basin. The forests surround two big cities - Bydgoszcz with 380 thousand residents and Torun with 210 thousand residents, as well as Solec Kujawski a smaller town situated between the two other ones. The Forest area comes to 44,000 ha. Forests are included within the forest inspectorates of Cierpiszewo, Gniewkowo, Solec and Bydgoszcz. Forests of the Torun Basin on the right bank of the Vistula River are included within the forest inspectorates of Dobrzejewice, Torun and Żołędowo. Inland dunes are a significant physiographic element of the valley. They are one of the most interesting inland dunes in Europe (Galon 1958; Mrózek 1958). One of them is the Zadroże Dune located by the administrative boundary of Torun. In the 1940s, it was one of the first research areas for ecologists from the Nicolaus Copernicus University created in Torun in 1945 (Nienartowicz et al. 2010).

Fires frequently occur in the Bydgoszcz Forest. a considerable area is covered with forests reconstructed on heaths. The largest post-fire area is located in the forest divisions of Cierpiszewo and Gniewkowo. The area was afforested after the great fire in August 1992, when almost 3,000 ha of forest burned down. a significant element of the Torun Forest and the Torun Basin is the military train- ing ground that has existed here for more than 300 years. It is overgrown with extensive heaths, which persist as a nonforest formation due to military activities and accompanying explosions of hand grenades and artillery shells, and fires initiated by them. Heaths occur also in areas that belong to industrial plants. One of the largest heathlands is situated near the poultry plants „Drosed” (operating until 2008) in the south-western frontiers of Torun, bordering on the Forest District and the village of Glinki.

Details concerning particular fragments of the Torun Basin are provided in papers included in the 12th Issue of the journal Ecological Questions. The course of boundaries of the Tuchola Forest region and its major areas subject to legal protection are presented in the paper by Jarzębski et al. (2010) included in the 13th Issue of EQ.

\section{Method}

\subsection{Collection of the material in the field}

For palynological analysis, samples were collected at 26 research sites, including 12 in the Tuchola Forest and 14 
in the Torun Basin, and more precisely - in the Bydgoszcz Forest. Within the first area, six sites were selected in the heaths; three in young pine growths adjacent to heaths and three in mature pine forests bordering on young growths, and thus situated a bit further from the heaths. In the Torun Basin, five sites were selected in heaths and five in mature pine stands. In young pine and birch growths, 4 sites were selected. At each site a relevé was made with the BraunBlanquet method, by making a list of plant species and by estimating their cover-abundance in forest layers $a_{1}, a_{2}, b$ and c. Only vascular plants were included in relevés. The surface area of relevés was usually $400 \mathrm{~m}^{2}$. Exceptionally in the case of narrow forest subsections, the area of relevés was smaller (ca. $300 \mathrm{~m}^{2}$, the surface area of a rectangle 15 x $20 \mathrm{~m}$ ). In the middle of each relevé, samples for palynological analyses were collected. Location of relevés and sample collection sites for palynological analysis were determined with the GPS receiver.

Samples for palynological analysis were collected in places overgrown with moss by means of a plastic cylinder with a diameter of $5 \mathrm{~cm}$ (the intersection area of $\left.19.6 \mathrm{~cm}^{2}\right)$.

The list of codes and descriptions of sites are presented in Table 1 .

\subsection{Preparation of samples for palynological analysis}

Samples collected in the field for palynological studies were transported to a laboratory, where they were cleaned off dead matter. Next, each sample was boiled in $10 \%$ $\mathrm{KOH}$ solution. After boiling-out, samples were filtered out and rinsed with distilled water, and then two tablets of Lycopodium were added into the thus-prepared liquid as an indicator. Next, the acetolysis of Erdtman was carried out on the studied samples in accordance with the recommendations of Berglund and Ralska-Jasiewiczowa (1986).

Each sample was analysed under a microscope. The number of vascular plant taxa and the percentage of pollen grains of particular taxa per sample were calculated based on the results of pollen analysis. Among bryophytes, only spores of Sphagnum were included in the analysis.

The formula of Stockmarr (1971) was applied in the calculation of pollen grains deposition on $1 \mathrm{~cm}^{2}$. With the software POLPAL (Nalepka \& Walanus 2003; Walanus \& Nalepka 1999), the so-called rarefaction index of species richness $\mathrm{E}(\mathrm{Sn})$ was also calculated for pollen samples according to the method proposed by Birks et al. (1988). This index indicates the number of pollen types occurring in samples with the same number of sporomorphs, because

Table 1. Symbols, location and syntaxonomic rank of relevés made at research sites, as well as other subsets of relevés included in numerical analyses

\begin{tabular}{|c|c|c|c|}
\hline $\begin{array}{l}\text { Site } \\
\text { No. }\end{array}$ & $\begin{array}{l}\text { Symbols } \\
\text { of relevés } \\
\text { and pollen } \\
\text { samples }\end{array}$ & Region, location & $\begin{array}{l}\text { Syntaxonomic rank, } \\
\text { vegetation types }\end{array}$ \\
\hline 1 & A-L1-Y & $\begin{array}{l}\text { Tuchola Forest, the forest complex along the road } \\
\text { Lubnia - Wiele }\end{array}$ & $\begin{array}{l}\text { pine plantation with a high contribution of Calluna } \\
\text { vulgaris }\end{array}$ \\
\hline 2 & A-L2-Y & as above & young birch - pine growth \\
\hline 3 & A-L3-O & as above & $\begin{array}{l}\text { old - growth pine forest, the association Leucobryo- } \\
\text { Pinetum, the variant with Arctostaphylos uva-ursi }\end{array}$ \\
\hline 4 & A-P1-N & $\begin{array}{l}\text { Tuchola Forest, the national park, the dune by the } \\
\text { section line }\end{array}$ & psammophilous grassland / heath \\
\hline 5 & A-P1-Y & $\begin{array}{l}\text { Tuchola Forest, firebreak by the section line at the } \\
\text { national park's boundary }\end{array}$ & young birch - pine growth \\
\hline 6 & A-P1-O & $\begin{array}{l}\text { Tuchola Forest, the national park, forest, ca. } 60 \mathrm{~m} \\
\text { from the previous relevé in the E direction }\end{array}$ & $\begin{array}{l}\text { old - growth pine forest, the associahce, Leucobryo- } \\
\text { Pinetum }\end{array}$ \\
\hline 7 & A-P2-N & $\begin{array}{l}\text { Tuchola Forest, the park, power and section lines at } \\
\text { the National Park's boundary }\end{array}$ & heath \\
\hline 8 & A-P2-O & $\begin{array}{l}\text { Tuchola Forest, the national park, forest, old-growth } \\
\text { pine forest, ca. } 60 \mathrm{~m} \text { from the previous relevé in the } \\
\text { E direction }\end{array}$ & Cladonio-Pinetum \\
\hline 9 & A-R1-N & $\begin{array}{l}\text { Tuchola Forest, to the south-east of the village of } \\
\text { Rolbik, the former airstrip area }\end{array}$ & heath \\
\hline
\end{tabular}


Table 1. Cont.

\begin{tabular}{|c|c|c|c|}
\hline $\begin{array}{l}\text { Site } \\
\text { No. }\end{array}$ & $\begin{array}{l}\text { Symbols } \\
\text { of relevés } \\
\text { and pollen } \\
\text { samples }\end{array}$ & Region, location & $\begin{array}{l}\text { Syntaxonomic rank, } \\
\text { vegetation types }\end{array}$ \\
\hline 10 & A-R1-Y & as above - forest plantations & young - pine growth directly adjacent to the heath \\
\hline 11 & A-R1-O & $\begin{array}{l}\text { Tuchola Forest, surroundings of the Rolbik village, } \\
\text { forest, old-growth pine forest in the vicinity of the } \\
\text { former airstrip }\end{array}$ & $\begin{array}{l}\text { old - growth pine forest, the association Leucobryo- } \\
\text { Pinetum }\end{array}$ \\
\hline 12 & A-T1-N & $\begin{array}{l}\text { Tuchola Forest, the Tuchola Forest Inspectorate, the } \\
\text { former airstrip area }\end{array}$ & heath \\
\hline 13 & A-T1-Y & as above & young birch - pine growth near the heath \\
\hline 14 & A-T1-O & $\begin{array}{l}\text { Tuchola Forest, the Tuchola Forest Inspectorate, } \\
\text { forest near the former airstrip }\end{array}$ & $\begin{array}{l}\text { old - growth pine forest, the association Leucobryo- } \\
\text { Pinetum }\end{array}$ \\
\hline 15 & B-C1-Y & $\begin{array}{l}\text { the Torun Basin, the commune of Wielka } \\
\text { Nieszawka, the Forest Inspectorate of Cierpiszewo, } \\
\text { surroundings of the Cierpice village, post-fire area, } \\
\text { regeneration of forest near the section line }\end{array}$ & $\begin{array}{l}\text { young birch growth with a high contribution of } \\
\text { Calluna vulgaris in the herb layer }\end{array}$ \\
\hline 16 & $\mathrm{~B}-\mathrm{C} 2-\mathrm{Y}$ & $\begin{array}{l}\text { the Torun Basin, the commune of Wielka } \\
\text { Nieszawka, the Forest Inspectorate of Cierpiszewo, } \\
\text { the vicinity of the Cierpice village, post-fire area, } \\
\text { regeneration of forest near the section line }\end{array}$ & young - pine growth \\
\hline 17 & B-G1-N & $\begin{array}{l}\text { the Torun Basin, Glinki (the district of Toruń), the } \\
\text { area near the poultry plants „Drosed" }\end{array}$ & $\begin{array}{l}\text { a patch of kinnikinnick bearberry in the phytocoenosis } \\
\text { dominated by Corynephorus canescens, lichens and } \\
\text { Polytrichum piliferum }\end{array}$ \\
\hline 18 & B-G2-N & $\begin{array}{l}\text { as above, the area disturbed by the construction of } \\
\text { the Torun ring road }\end{array}$ & the heath Arctostaphylo-Callunetum \\
\hline 19 & B-G3-N & as above, near the forest & $\begin{array}{l}\text { a patch of kinnikinnick bearberry in the phytocoenosis } \\
\text { dominated by Calluna vulgaris, Pleurozium schreberi } \\
\text { and Pinus sylvestris in the layer b }\end{array}$ \\
\hline 21 & B-M1-N & $\begin{array}{l}\text { the Torun Basin, the commune of Wielka } \\
\text { Nieszawka, the village of Mała Nieszawka, the } \\
\text { Forest Inspectorate of Cierpiszewo, the power line }\end{array}$ & heath \\
\hline 22 & B-M1-Y & $\begin{array}{l}\text { the Torun Basin, the commune of Wielka } \\
\text { Nieszawka, the village of Mała Nieszawka, the } \\
\text { Forest Inspectorate of Cierpiszewo, pine forest, ca. } \\
50 \mathrm{~m} \text { from the previous site in the W direction }\end{array}$ & pine pole wood \\
\hline 20 & B-M2-N & $\begin{array}{l}\text { the Torun Basin, the commune of Wielka } \\
\text { Nieszawka, the wartime cemetery near the Zadroże } \\
\text { Dune }\end{array}$ & xerothermic grassland \\
\hline 23 & $\mathrm{~B}-\mathrm{Z} 1-\mathrm{N}$ & $\begin{array}{l}\text { the Torun Basin, the commune of Wielka } \\
\text { Nieszawka, the Zadroże Dune, the forest's edge, the } \\
\text { escarpment with } N \text { exposure }\end{array}$ & heath \\
\hline 24 & $\mathrm{~B}-\mathrm{Z2}-\mathrm{O}$ & $\begin{array}{l}\text { the Torun Basin, the commune of Wielka } \\
\text { Nieszawka, the Zadroże Dune, forest, pine forest } \\
\text { stand ca. } 50 \text {-year-old }\end{array}$ & young - pine forest \\
\hline 25 & B-Z3-O & as above & young - pine forest with Padus serotina \\
\hline 26 & B-Z4-O & $\begin{array}{l}\text { the Torun Basin, the commune of Wielka } \\
\text { Nieszawka, the Zadroże Dune, the forest's edge } \\
\text { next to the wartime cemetery }\end{array}$ & $\begin{array}{l}\text { young - pine forest with Robinia pseudacacia; } \\
\text { Corynephorus canescens and Spergula vernalis occur } \\
\text { in the layer c; in the layer d, besides mosses, also } \\
\text { lichens occur abundantly }\end{array}$ \\
\hline
\end{tabular}


the rarefaction analysis reduces all the samples to the same total number of pollen grains. This method permits the comparison between samples differing from each other in the amount of counted sporomorphs.

\subsection{Numerical analysis of phytosociological and palynological data, as well as the assessment of species richness and species diversity}

In order to define the variability within the set of relevés, as well as to identify what are the relationships between the structure of plant communities and the pollen rain, a separate hierarchical accumulative classification was performed on one phytosociological data set and two palynological data sets (a table with percentage structure of modern pollen rain and a table with deposition of sporomorphs of particular taxa per $1 \mathrm{~cm}^{2}$ ). The aforementioned data were compiled in Microsoft Excel tables and then used in the software MVSP PLUS version 3.1 (Kovach 1985-1999), with which the hierarchical accumulative classification was performed, applying the average linkage clustering method (Orlóci 1978). Also the ordination of surface samples was performed in terms of types and concentration of pollen types with the method of principal component analysis (PCA). Logarithmic transformation $\left(\log _{10}\right)$ of the data was performed before the ordination.

Also the Shannon diversity index and the evenness index of relevés and palynological samples were calculated with the software MVSP v. 3.1. In the Shannon formula, the logarithm base 2 was applied. When entering the phytosociological data into Microsoft Excel tables, coefficients of species density or species cover in each relevé were converted from the Braun-Blanquet scale into their numerical equivalents of the scale by Jansen (1975) and van der Maarel (1979) in the following way: $\mathrm{r}-1,+-2,1-3,2-$ $5,3-7,4-8,5-9$. Then, the cover coefficients expressed in the new scale were multiplied by numbers expressing the average height of particular layers in a plant community. They had the following values: 1 for the layer c, 5 for the layer $b, 15$ for the layer $a_{2}$ and 20 for the layer $a_{1}$. The additional coefficients applied for particular layers aimed at emphasizing the differences between non-forest and forest communities in the sets of phytosociological data.

\subsection{Assessment of the significance of differences between pollen samples and the analysis of pollen - vegetation relationship}

To evaluate the homogeneity of variance for pollen samples, the Levine test was applied. Due to heterogeneity of variance, the evaluation of the significance of differences was performed with the nonparametric Kruskal-Wallis test. Also values of Spearman correlation coefficients were determined between the abundance of some species in in- dividual relevés and the concentration of their pollen in relevant pollen samples, as well as between a value of the diversity index for a phytocoenosis, calculated with the Shannon formula and the concentration of some pollen types. The software PAST was applied in the calculations (Hammer et al. 2001).

Indices of association (A), under-representation (U) and over-representation $(\mathrm{O})$ were calculated in accordance with Davis (1984) and Fontana (2005), using the presence - absence data of a plant taxon and its pollen from relevés. The indices were calculated by the following formulae for all taxa found both as a plant and pollen in one or more stands:

$$
\begin{aligned}
& \mathrm{A}=\mathrm{B}_{0}\left(\mathrm{P}_{0}+\mathrm{P}_{1}+\mathrm{B}_{0}\right)^{-1} \\
& \mathrm{U}=\mathrm{P}_{1}\left(\mathrm{P}_{1}+\mathrm{B}_{0}\right)^{-1} \\
& \mathrm{O}=\mathrm{P}_{0}\left(\mathrm{P}_{0}+\mathrm{B}_{0}\right)^{-1}
\end{aligned}
$$

where $\mathrm{B}_{0}$ is the number of stands in which the pollen type is present in the surface sample and the associated plant taxon is present in the vegetation; $\mathrm{P}_{0}$ is the number of stands where the pollen type is present in the surface sample but the associated plant taxon is not present in the vegetation, and $P_{1}$ is the number of stands where the plant taxon is present in the vegetation but the pollen type is not present in the surface sample.

\section{Results}

\subsection{Species composition and numerical classification of relevés}

A total of 60 taxa of vascular plants occurred in 26 relevés, including 11 tree species, 4 species of shrubs, 4 dwarf shrubs and 41 herbaceous species (Table 2). In the layer $a_{1}$ of eight relevés made in mature forests, two species of trees were recorded, i.e. Pinus sylvestris and Betula pendula. In the layer $\mathrm{a}_{2}$ of these relevés, apart from the two aforementioned species, also the presence of black cherry Padus serotina was recorded. This species, coming from North America, spread abundantly in forests of the Torun Basin. In the layer $b$ of the whole set of relevés, 5 tree species and two shrub species were recorded. Among trees in the analysed sites, Tilia cordata and Berberis vulgaris occurred only in this forest layer. In three subsets of relevés - non-forest communities, young growths and mature forests - many common species occur. Taking into account only the species composition, a high similarity between relevés becomes pronounced. When taking into account the abundance of phytocoenoses' components, the species occurring in layers $a_{1}$ and $a_{2}$ are the most differentiating characteristics of the three studied subsets of relevés.

Based on numerical classification of relevés, it was found that relevés made in mature forests are considerably different. They form a separate homogeneous group of ob- 
Table 2. Species composition and their abundance after transformations in 26 relevés

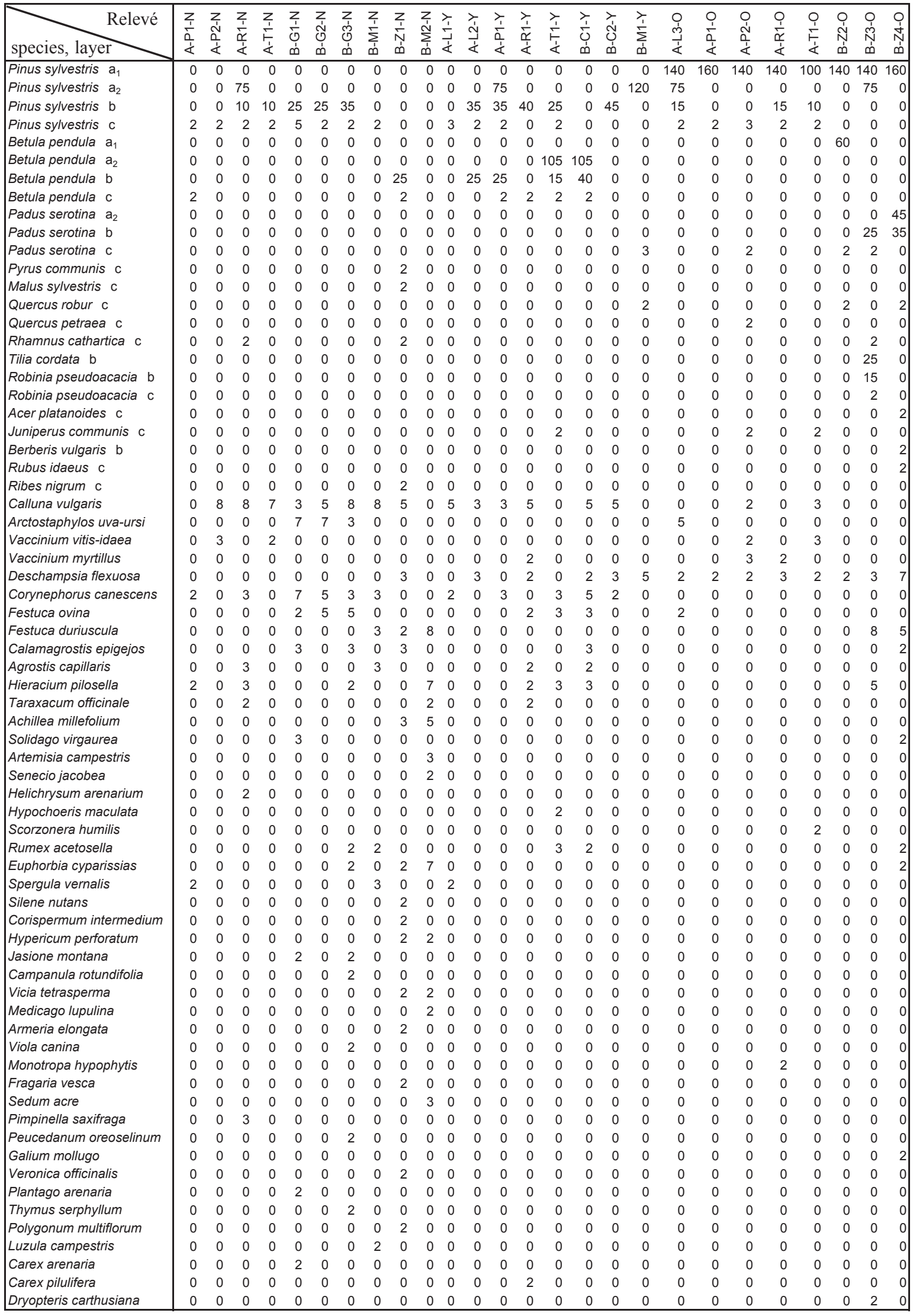




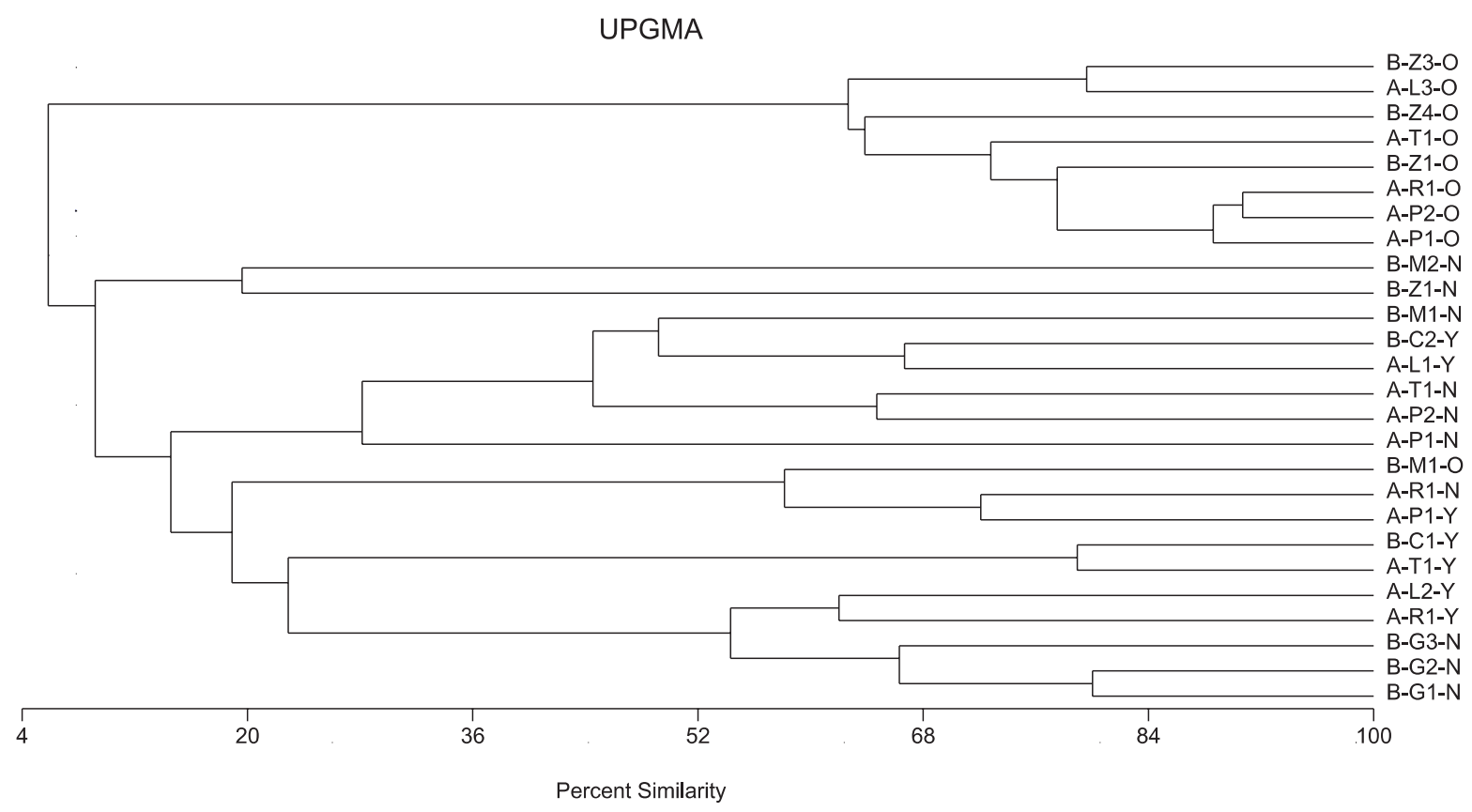

Figure 2. Dendrogram of 26 relevés based on the species composition and their quantitative variation (clustered with UPGMA and the percentage similarity index)

jects in the dendrogram and the similarities between them exceed $60 \%$ (Fig. 2). Among other samples, only small clusters, formed by 2-3 relevés, are being distinguished at the similarity level above $60 \%$. Some of these groups are also homogeneous, as they include only samples made in non-forest communities $(\mathrm{N})$ or in young forests $(\mathrm{Y})$. These are e.g. the cluster of three relevés made in the heaths with Arctostaphylos uva-ursi in the city district Glinki (three relevés denoted as B-G1-N, B-G2-N and B-G3-N), the cluster of two relevés made in the heaths in Tuchola Forest (A-P2-N and A-T1-N), as well as two clusters, each of them consisting of two relevés from young forest phytocoenoses made in both regions (Fig. 2, the cluster comprising relevés $\mathrm{A}-\mathrm{L} 1-\mathrm{Y}$ and $\mathrm{B}-\mathrm{C} 2-\mathrm{Y}$, as well as the group of objects A-T1-Y and B-C1-Y). Some of these small groups include, however, both relevés made in non-forest communities, as well as in young forests (e.g. Fig. 2, the group consisting of A-R1-N and A-P1-Y).

At the higher fusion levels of the dendrogram, corresponding to low percentage of similarity, almost exclusively heterogeneous groups occur. The group of two relevés made in non-forest communities on the edge of the Zadroże Dune is the only exception (relevés denoted as B-M2-N and B-Z1-N). Both relevés, however, differ considerably in the species composition. Their similarity amounts to just ca. 20\% (Fig. 2). Relevé B-M2-N made on the wartime cemetery is the only element of the analysed set in which species, such as Festuca duriuscula, Sedum acre, Artemisia campestris, Medicago lupulina, Achillea millefolium, Taraxacum officinale, Vicia tetrasperma and Senecio jacobea occur quite abundantly. The presence of the aforementioned characteristics causes that the similarity between relevé B-M2-N and other relevés is low.

\subsection{The count, the composition and the taxonomic diversity of pollen surface samples}

56 pollen taxa were recorded in 26 surface samples (Table 3). In all samples, pollen of Pinus, Betula and Alnus were being observed (Fig. 3). In nearly all samples, pollen of Corylus occurred (in 24 samples out of 26), as well as Ericaceae (25/26) and poaceae (23/26). Pollen of Artemisia (22/26), Calluna (21/26) and Cerealia (20/26) occurred in large quantities.

Samples differed considerably in terms of pollen abundance. The highest deposition of sporomorphs, i.e. as many as 32,043 grains $/ \mathrm{cm}^{2}$, was recorded in a pollen sample at site A-P2-O, i.e. in the mature pine forest in the habitat of dry coniferous forest at the boundary of the Tuchola Forest National Park. The quantity of pollen of Pinus $(26,933$ grains $\left./ \mathrm{cm}^{2}\right)$ and Alnus $\left(1,683\right.$ grains $\left./ \mathrm{cm}^{2}\right)$ recorded in that sample was the highest among all the sites. Also the sample collected at site B-M2-N, which is distinguished by the vegetation species composition, was characterized by high density - 19,308 pollen grains $/ \mathrm{cm}^{2}$ recorded. When comparing all the samples, the largest quantity of Corylus and Tilia pollen occurred at that site, as well as pollen of Artemisia, Rumex acetosella/acetosa t. and Cerealia-t. It 


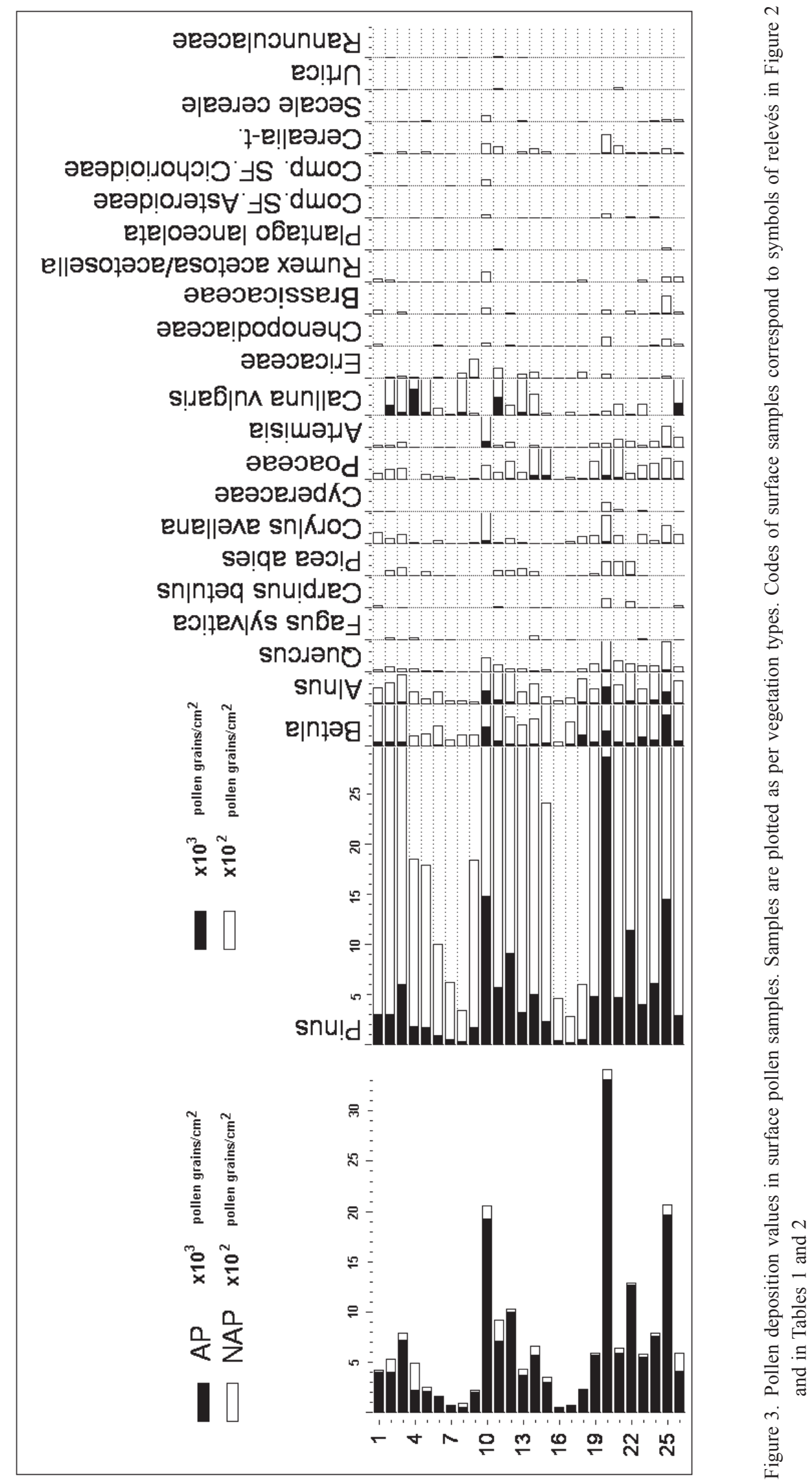




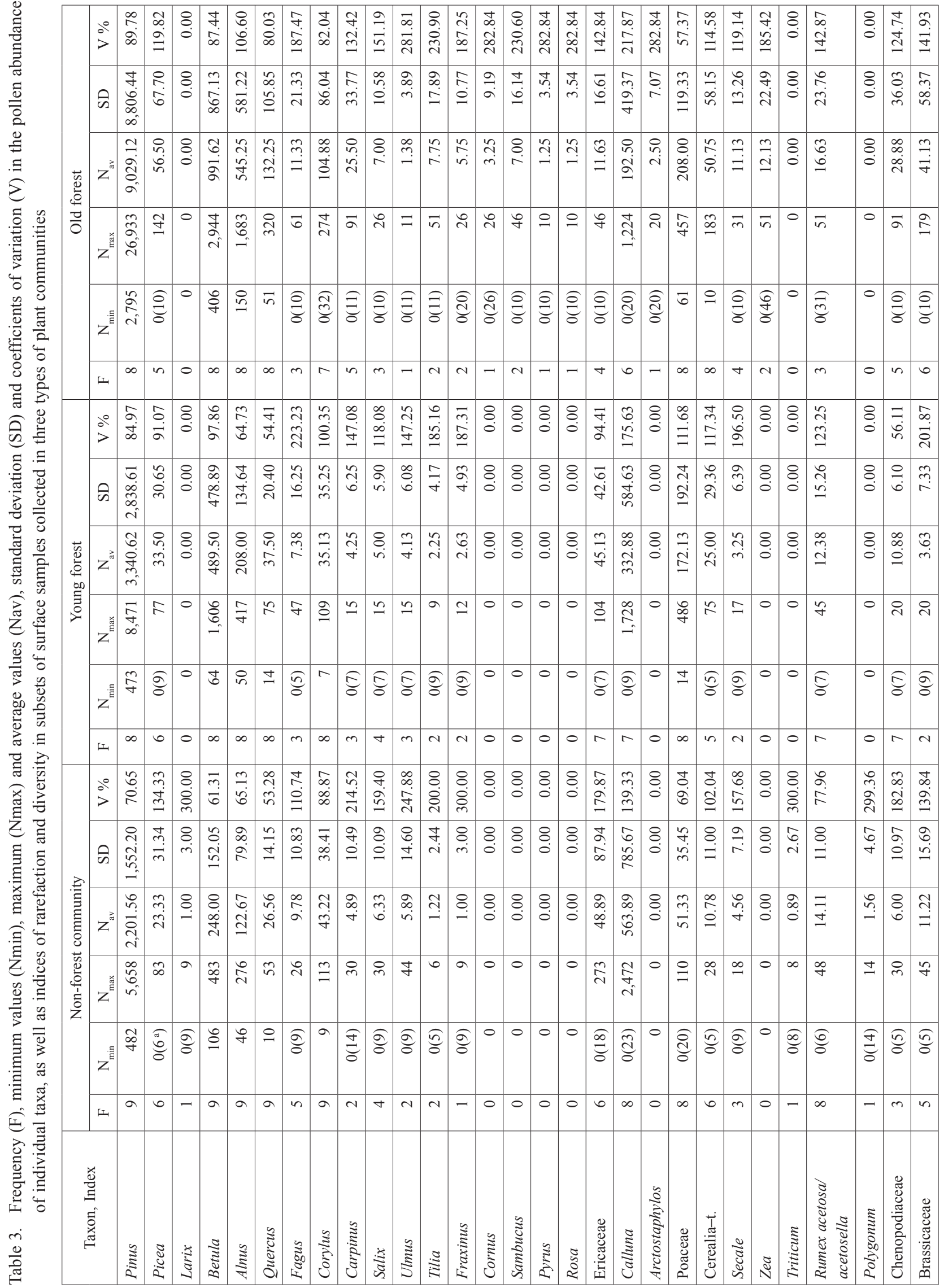




\begin{tabular}{|c|c|c|c|c|c|c|c|c|c|c|c|c|c|c|c|c|c|c|c|c|c|c|c|c|c|c|c|}
\hline \multirow{6}{*}{$\begin{array}{l}\overrightarrow{v_{0}} \\
\stackrel{0}{0} \\
\overrightarrow{0} \\
0\end{array}$} & 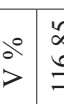 & $\begin{array}{l}\infty \\
\infty \\
\stackrel{\infty}{=} \\
\stackrel{n}{\prime}\end{array}$ & $\stackrel{8}{\circ}$ & \begin{tabular}{|l|}
$\vec{R}$ \\
$\dot{\alpha}$ \\
0
\end{tabular} & $\stackrel{8}{8}$ & \begin{tabular}{|c|} 
\\
$\stackrel{m}{0}$ \\
$\stackrel{0}{0}$ \\
-
\end{tabular} & 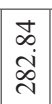 & 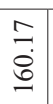 & 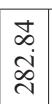 & $\begin{array}{l}\mathcal{I} \\
\stackrel{d}{0} \\
\stackrel{1}{d}\end{array}$ & 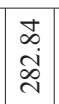 & 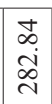 & $\begin{array}{l}\mathcal{I} \\
i \\
\text { D } \\
i\end{array}$ & $\stackrel{8}{0}$ & 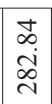 & 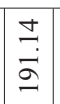 & $\stackrel{8}{8}$ & 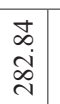 & $\begin{array}{l}8 \\
0\end{array}$ & 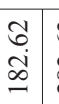 & 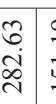 & $\stackrel{\circ}{\overrightarrow{\vec{n}}}$ & $\mid$\begin{tabular}{c|}
$\dot{B}$ \\
$\dot{\infty}$ \\
$\dot{\infty}$
\end{tabular} & $\begin{array}{l}\text { ते } \\
\text { స్}\end{array}$ & 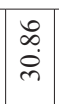 & $\begin{array}{l}\hat{T} \\
\stackrel{\sim}{\sim}\end{array}$ & $\mid \begin{array}{l}\hat{n} \\
\infty \\
\infty \\
-1\end{array}$ \\
\hline & की & $\begin{array}{l}\infty \\
\stackrel{\infty}{n} \\
\end{array}$ & 8 & $\begin{array}{l}\hat{a} \\
\dot{b} \\
\dot{n}\end{array}$ & $\stackrel{8}{\circ}$ & $\stackrel{\ominus}{\stackrel{m}{\dot{m}}}$ & $\frac{\partial}{a}$ & f̆ & $\stackrel{\vdots}{i}$ & $\hat{\tilde{m}}$ & $\mid \begin{array}{c}\vec{w} \\
m \\
m\end{array}$ & 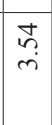 & $\begin{array}{l}n \\
n \\
a\end{array}$ & $\stackrel{8}{0}$ & $\begin{array}{l}\text { int } \\
\text { in }\end{array}$ & $\mid \begin{array}{l}\vec{b} \\
\dot{b}\end{array}$ & $\stackrel{8}{\circ}$ & 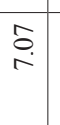 & $\begin{array}{l}8 \\
\vdots \\
\dot{0}\end{array}$ & $\begin{array}{l}\stackrel{0}{0} \\
\stackrel{\infty}{0}\end{array}$ & 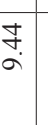 & 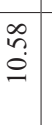 & 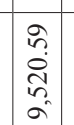 & $\vec{m}$ & $\mid \begin{array}{l}0 \\
0 \\
0\end{array}$ & $\stackrel{\circ}{\circ}$ & $\stackrel{\overbrace{}}{i}$ \\
\hline & $z^{z}$ & 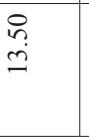 & $\stackrel{8}{0}$ & $\begin{array}{l}8 \\
0 \\
\dot{\infty}\end{array}$ & 8 & $\begin{array}{l}0 \\
0 \\
0 \\
0\end{array}$ & $\begin{array}{l}\tilde{y} \\
\tilde{m}\end{array}$ & $\begin{array}{l}\infty \\
\infty \\
\infty \\
\infty \\
\infty\end{array}$ & $\begin{array}{l}n \\
\text { in } \\
i\end{array}$ & $\begin{array}{l}\stackrel{i}{n} \\
+\end{array}$ & $\stackrel{\overbrace{}}{\rightarrow}$ & $\stackrel{2}{\longrightarrow}$ & 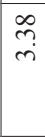 & $\stackrel{8}{0}$ & $\stackrel{\text { I }}{\rightarrow}$ & $\begin{array}{c}\infty \\
\infty \\
\infty \\
\infty\end{array}$ & $\stackrel{8}{\circ}$ & $\begin{array}{l}\stackrel{D}{n} \\
i\end{array}$ & $\stackrel{8}{\circ}$ & 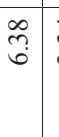 & 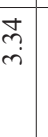 & $\underset{\sim}{\stackrel{8}{0}}$ & 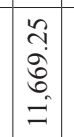 & $\begin{array}{l}0 \\
i \\
\infty \\
\infty\end{array}$ & $\stackrel{g}{i}$ & 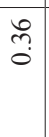 & 苛 \\
\hline & $z^{\frac{a}{2}}$ & + & 0 & 亗 & 0 & $\vec{\sigma}$ & i & i & iి & $i$ & 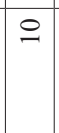 & $\stackrel{9}{ }$ & $\hat{\lambda}$ & 0 & 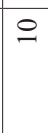 & $\vec{\gamma}$ & 0 & i & 0 & $\vec{n}$ & $\hat{\imath}$ & $\underset{\pi}{\pi}$ & $\mid \begin{array}{c}\delta \\
\dot{0} \\
\dot{0} \\
2 \\
\sigma \\
-1\end{array}$ & $\begin{array}{l}\stackrel{8}{+} \\
\text { d }\end{array}$ & $\left|\begin{array}{c}\infty \\
i \\
i\end{array}\right|$ & $\tilde{3}$ & $\stackrel{\circ}{\stackrel{9}{-}}$ \\
\hline & $z^{2}$ & $\stackrel{\varrho}{\overparen{\sigma}}$ & 0 & $\vec{m}$ & 0 & 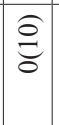 & 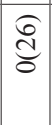 & $\stackrel{\widehat{O}}{\sigma}$ & 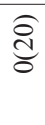 & $\stackrel{\varrho}{\Xi}$ & $\stackrel{\widehat{\Xi}}{\Xi}$ & $\stackrel{\varrho}{\stackrel{\theta}{\sigma}}$ & 离 & 0 & $\stackrel{\widehat{\theta}}{\sigma}$ & \begin{tabular}{|c|} 
\\
$d$ \\
$d$ \\
$\sigma$
\end{tabular} & 0 & 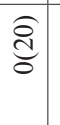 & 0 & $\widehat{\vec{\sigma}}$ & $\widehat{d}$ & $\stackrel{\widehat{\theta}}{\mathrm{g}}$ & $\begin{array}{l}8 \\
0 \\
0 \\
n \\
n \\
n\end{array}$ & 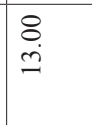 & $\bar{g}$ & ָุ & $\stackrel{\rho}{\infty}$ \\
\hline & I & in & 0 & $\infty$ & 0 & $\sigma$ & - & $m$ & - & $\sim$ & - & -7 & - & 0 & - & $N$ & 0 & - & 0 & - & - & $m$ & $\infty$ & $\infty$ & $\infty$ & $\infty$ & $\infty$ \\
\hline \multirow{6}{*}{ 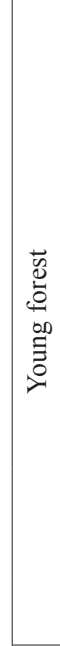 } & $\begin{array}{l}\circ \\
>\end{array}$ & 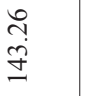 & 8 & $\begin{array}{l}9 \\
\stackrel{9}{.} \\
\stackrel{5}{0}\end{array}$ & $\stackrel{8}{\circ}$ & 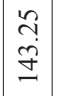 & \begin{tabular}{|c|}
0 \\
$\stackrel{0}{i}$ \\
$\tilde{d}$ \\
$\stackrel{\sim}{c}$
\end{tabular} & $\begin{array}{l}\stackrel{i}{:} \\
\stackrel{\vec{n}}{\vec{v}}\end{array}$ & $\stackrel{8}{\circ}$ & $\stackrel{8}{0}$ & \begin{tabular}{|c|} 
\\
$\stackrel{n}{?}$ \\
$\substack{\infty \\
\sim}$
\end{tabular} & $\stackrel{8}{0}$ & $\begin{array}{l}\infty \\
\stackrel{\infty}{i} \\
\infty \\
\sim \\
\sim\end{array}$ & 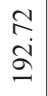 & 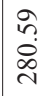 & $\stackrel{8}{\circ}$ & $\stackrel{8}{\circ}$ & $\stackrel{8}{\circ}$ & $\stackrel{8}{\circ}$ & $\begin{array}{c}m \\
0 \\
\dot{j} \\
\infty \\
\sim \\
-1\end{array}$ & \&. & $\begin{array}{l}\text { तิ } \\
\text { }\end{array}$ & $\mid$\begin{tabular}{c|}
0 \\
$?$ \\
0 \\
0 \\
0
\end{tabular} & $\begin{array}{l}\stackrel{g}{0} \\
\text { ف }\end{array}$ & \begin{tabular}{l|}
$\infty$ \\
$\tilde{i}$ \\
$\dot{i}$ \\
\end{tabular} & $\begin{array}{l}\tilde{a} \\
\dot{d} \\
\dot{d}\end{array}$ & $\stackrel{\infty}{\stackrel{\infty}{=}}$ \\
\hline & 家 & $\begin{array}{l}\vec{\sigma} \\
\dot{r}\end{array}$ & $\stackrel{8}{\circ}$ & $\begin{array}{l}n \\
\hat{n} \\
\text { no }\end{array}$ & $\begin{array}{l}8 \\
0\end{array}$ & $\overrightarrow{\dot{b}}$ & $\begin{array}{l}0 \\
i \\
i n \\
n\end{array}$ & $\stackrel{n}{\rightarrow}$ & $\stackrel{8}{\circ}$ & 8 & $\stackrel{\infty}{m}$ & $\stackrel{8}{0}$ & $\begin{array}{l}\text { Dे } \\
\text { in }\end{array}$ & $\begin{array}{l}\infty \\
\stackrel{\infty}{n}\end{array}$ & $\stackrel{\text { I }}{-}$ & $\begin{array}{l}8 \\
0 \\
0\end{array}$ & $\stackrel{8}{\circ}$ & $\stackrel{8}{\circ}$ & $\stackrel{8}{0}$ & 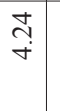 & 8 & 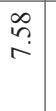 & 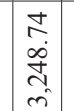 & $\hat{i}$ & $\vec{n}$ & $\stackrel{7}{0}$ & $\underset{+}{+}$ \\
\hline & $z=$ & $\begin{array}{l}\stackrel{n}{n} \\
m \\
m\end{array}$ & 8 & $\begin{array}{l}7 \\
9\end{array}$ & $\begin{array}{l}8 \\
0\end{array}$ & $\begin{array}{l}n \\
n \\
m\end{array}$ & \begin{tabular}{|c|}
$\infty$ \\
$\infty$ \\
-
\end{tabular} & 亦 & 8 & 8 & $\stackrel{M}{=}$ & 8 & $\begin{array}{l}\infty \\
\infty \\
- \\
-\end{array}$ & $\underset{\dot{m}}{8}$ & : & $\begin{array}{l}8 \\
0\end{array}$ & $\stackrel{8}{\circ}$ & 8 & 8 & $\stackrel{\text { n̊ }}{\rightarrow}$ & 8 & $\stackrel{\infty}{\stackrel{\infty}{\sim}}$ & 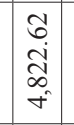 & 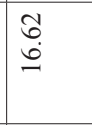 & $\underset{i}{i}$ & $\begin{array}{l}7 \\
0 \\
0\end{array}$ & సે \\
\hline & $z^{\underline{a}}$ & $\simeq$ & 0 & in & 0 & $\simeq$ & $\because$ & i & 0 & 0 & $a$ & 0 & $\because$ & $\because$ & in & 0 & 0 & 0 & 0 & $\simeq$ & 0 & $\stackrel{i}{ }$ & $\begin{array}{l}8 \\
i \\
i \\
\vdots \\
\sigma\end{array}$ & $\begin{array}{l}8 \\
\stackrel{1}{1}\end{array}$ & $\begin{array}{l}\tilde{S} \\
i\end{array}$ & $\begin{array}{l}0 \\
0 \\
0\end{array}$ & $\begin{array}{l}R \\
\stackrel{g}{2}\end{array}$ \\
\hline & $z^{2}$ & $E$ & 0 & $\widehat{\tilde{a}}$ & 0 & $E$ & $\frac{\pi}{\Xi}$ & 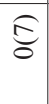 & 0 & 0 & $\widehat{\mathscr{\partial}}$ & 0 & $\frac{\pi}{0}$ & $\hat{\mathscr{g}}$ & $\widehat{\tilde{g}}$ & 0 & 0 & 0 & 0 & $\widehat{\mathbb{E}}$ & 0 & $E$ & \begin{tabular}{|c|} 
\\
$\dot{\vec{D}}$ \\
$\infty$
\end{tabular} & $\begin{array}{l}\stackrel{8}{\text { I }} \\
\text {. }\end{array}$ & \begin{tabular}{|c|} 
\\
$\infty$ \\
0 \\
0
\end{tabular} & 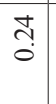 & : \\
\hline & I & $m$ & 0 & 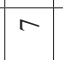 & 0 & $m$ & -7 & $N$ & 0 & 0 & - & 0 & - & $N$ & - & 0 & 0 & 0 & 0 & - & 0 & in & $\infty$ & $\infty$ & $\infty$ & $\infty$ & $\infty$ \\
\hline \multirow{6}{*}{$\exists$} & 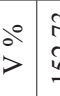 & 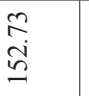 & $\begin{array}{l}\text { ते } \\
\text { ते } \\
\text { nd }\end{array}$ & 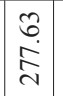 & 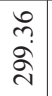 & $\begin{array}{c}\stackrel{m}{\infty} \\
\stackrel{2}{\sigma} \\
\stackrel{2}{\sigma}\end{array}$ & 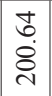 & $\begin{array}{l} \\
\\
\end{array}$ & 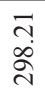 & \begin{tabular}{l}
0 \\
0 \\
\multirow{8}{*}{} \\
त.
\end{tabular} & \begin{tabular}{l|}
$\vec{y}$ \\
$\infty$ \\
$\alpha$ \\
$\grave{\lambda}$
\end{tabular} & \&. & 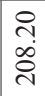 & $\begin{array}{l}\text { bे } \\
\dot{a} \\
\text {. }\end{array}$ & 8 & $\stackrel{8}{0}$ & $\begin{array}{l}8 \\
\dot{0} \\
\dot{0}\end{array}$ & 吕 & 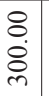 & $\stackrel{8}{\circ}$ & $\begin{array}{l}\text { ח़ं } \\
\text { aे }\end{array}$ & ते & $\begin{array}{l}\stackrel{0}{\infty} \\
\infty \\
\infty\end{array}$ & $\begin{array}{l}\vec{b} \\
\vec{d} \\
d\end{array}$ & \begin{tabular}{|c|}
$\hat{\infty}$ \\
$\dot{\vec{v}}$ \\
\end{tabular} & \begin{tabular}{|l|}
$\infty$ \\
$\stackrel{0}{0}$ \\
$\stackrel{2}{2}$
\end{tabular} & $\begin{array}{l}q \\
q \\
\infty \\
\infty\end{array}$ \\
\hline & हि & $\overrightarrow{\vec{m}}$ & $\begin{array}{l}\stackrel{?}{+} \\
\stackrel{+}{*}\end{array}$ & $\begin{array}{l}0 \\
\check{z} \\
y\end{array}$ & $\begin{array}{l}\tilde{\sigma} \\
+ \\
+\end{array}$ & $\begin{array}{l}\stackrel{0}{2} \\
i \\
\end{array}$ & $\stackrel{m}{\vec{m}}$ & $\stackrel{8}{8}$ & $\stackrel{5}{-}$ & $\stackrel{f}{\tilde{g}}$ & $\stackrel{\leftarrow}{-}$ & $\stackrel{8}{0}$ & $\begin{array}{l}\infty \\
\stackrel{\infty}{i} \\
i\end{array}$ & I্ & 8 & 8 & $\underset{\dot{m}}{\stackrel{8}{ }}$ & $\begin{array}{l}8 \\
0 \\
0\end{array}$ & $\begin{array}{l}\vec{b} \\
i\end{array}$ & $\stackrel{8}{\circ}$ & $\begin{array}{l}\overleftarrow{\sigma} \\
+\end{array}$ & $\begin{array}{l}\text { ¿े } \\
\text { ì }\end{array}$ & $\mid \begin{array}{l}\tilde{m} \\
\dot{p} \\
\dot{0} \\
i\end{array}$ & $\underset{f}{\stackrel{\infty}{f}}$ & 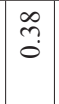 & \begin{tabular}{|l|}
0 \\
0 \\
0
\end{tabular} & $\bar{g}$ \\
\hline & $z=$ & 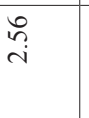 & $\overrightarrow{\vec{i}}$ & $\begin{array}{c}\tilde{m} \\
\\
\end{array}$ & $\stackrel{\leftrightarrow}{\sim}$ & 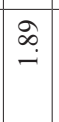 & 吕 & $\stackrel{8}{\circ}$ & مُ & $\bar{\sigma}$ & 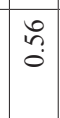 & $\stackrel{8}{8}$ & $\underset{i}{\stackrel{J}{i}}$ & 总 & $\stackrel{8}{\circ}$ & $\stackrel{8}{0}$ & $\stackrel{\leftrightarrow}{-}$ & $\begin{array}{l}8 \\
0 \\
0\end{array}$ & $\begin{array}{c}\stackrel{\infty}{\infty} \\
\stackrel{0}{0}\end{array}$ & $\stackrel{8}{0}$ & 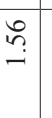 & $\underset{g}{J}$ & $\mid \begin{array}{c}a \\
\dot{a} \\
\dot{g} \\
\dot{f} \\
\hat{j}\end{array}$ & గై & $\begin{array}{l}\stackrel{0}{-} \\
-1\end{array}$ & J & 守 \\
\hline & $z^{2}$ & $a$ & $\Xi$ & in & \pm & $a$ & $\infty$ & 0 & in & $F$ & in & 0 & \pm & $a$ & 0 & 0 & $a$ & 0 & $\infty$ & 0 & I & $\infty$ & 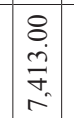 & $\begin{array}{l}\stackrel{8}{\text { d }} \\
\text { in }\end{array}$ & $\stackrel{\stackrel{+}{\mathrm{i}}}{\mathrm{i}}$ & in & $\begin{array}{l}8 \\
\dot{I}\end{array}$ \\
\hline & $z^{\underline{E}}$ & $\frac{\mathrm{g}}{\mathrm{g}}$ & $\sqrt{0}$ & $\tilde{a}$ & 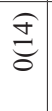 & $\widehat{\infty}$ & $\stackrel{6}{6}$ & 0 & $\frac{\tilde{E}}{8}$ & $\frac{\tilde{g}}{8}$ & $\sqrt{\sigma}$ & 0 & $\widehat{\infty}$ & $\frac{\hat{\sigma}}{8}$ & 0 & 0 & $\widehat{\mathscr{g}}$ & 0 & $\stackrel{\infty}{0}$ & 0 & $\underset{\Xi}{\stackrel{\vartheta}{\rightleftarrows}}$ & $\frac{\tilde{g}}{0}$ & $\begin{array}{l}8 \\
\dot{a} \\
2 \\
=\end{array}$ & $\underset{\sigma}{8}$ & $\stackrel{\tilde{n}}{n}$ & $\begin{array}{l}0 \\
\tilde{c} \\
0\end{array}$ & $\stackrel{8}{\circ}$ \\
\hline & \begin{tabular}{l|l}
$I$ &
\end{tabular} & $m$ & $N$ & 0 & - & $N$ & $\sim$ & 0 & - & $m$ & - & 0 & $\alpha$ & $\alpha$ & 0 & 0 & - & 0 & - & 0 & - & $\sim$ & $a$ & $a$ & $a$ & $a$ & $\sigma$ \\
\hline 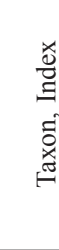 & & 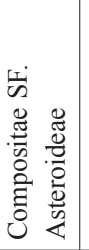 & 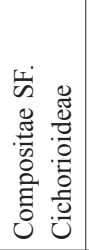 & 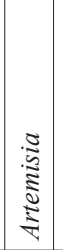 & $\begin{array}{l}\hat{U} \\
\bar{\Xi} \\
\bar{z}\end{array}$ & 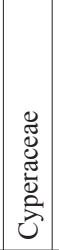 & 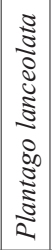 & 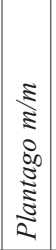 & $\approx$ & 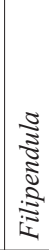 & 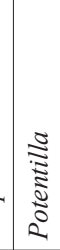 & & 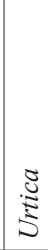 & 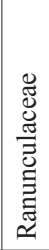 & 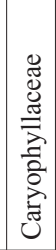 & 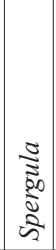 & 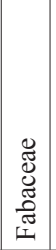 & 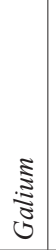 & 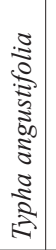 & 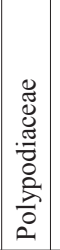 & & $\nu^{\pi}$ & 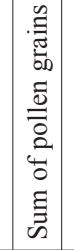 & 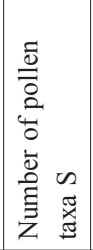 & 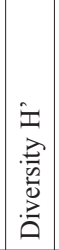 & 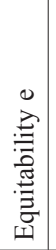 & 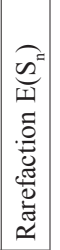 \\
\hline
\end{tabular}


was the only sample in which the pollen of Sorbus was recorded.

High deposition was also recorded at site A-L3-O near the village of Lubnia, i.e. in the pine forest with Arctostaphylos uva-ursi in the herb layer. It was the only sample in which pollen grains of the aforementioned dwarf shrub were observed.

The least amount of sporomorphs (821 pollen grains/ $\mathrm{cm}^{2}$ ) was recorded in sample B-M1-Y collected in the young growth near the heath developing under the power line in the village of Mała Nieszawka. Small deposition was recorded also at sites B-G3-N (1,195 grains/ $\left.\mathrm{cm}^{2}\right)$, BG2-N (1,376 grains/cm $\left.{ }^{2}\right)$ and B-C2-Y $(1,306$ grains/cm²).

On average, in 9 samples collected in non-forest communities (excluding sites B-M2-N on the wartime cemetery with considerably different vegetation) 3,449.89 pollen grains $/ \mathrm{cm}^{2}$ were recorded, in samples collected in young growths $-4,822.62$ grains $/ \mathrm{cm}^{2}$, and in old forests $11,669.25$ grains $/ \mathrm{cm}^{2}$.

The highest number of pollen types (24 of each type) occurred in old forests on the Zadroże Dune. Slightly less, 22 taxa occurred in a sample collected in the heath situated at the boundary with the Tuchola Forest National Park. 21 taxa occurred in sample A-T1-O, and 20 taxa occurred both in sample B-Z1-N (the heath on the Zadroże Dune), in sample B-M2-N (the wartime cemetery in the vicinity of this dune) and in the young pine wood near the village of Lubnia in Tuchola Forest). The fewest number of taxa (just 9) was recorded in sample B-M1-N in the heath under the power line in the village of Mała Nieszawka. Slightly more taxa were in the sample from the heath at the airstrip in the Forest Inspectorate of Tuchola (11 taxa) and in the heath under the power line in the village of Mała Nieszawka (12 taxa).

The average number of taxa in samples collected in non-forest communities (excluding site B-M2-N) amounted to 16.33 , in young stands -16.62 and in old forests -18.50 . Due to increasing pine domination in this series of communities, the species diversity index was decreasing, reaching the average values of $1.76,1.71$ and 1.49 respectively in non-forest communities, young growths and old forests (Table 3). The increased domination of pollen of certain taxa, especially trees, in the studied temporal series is reflected in the decreased evenness. In samples from the non-forest communities, young growths and old forests, its average value amounts to $0.44,0.42$ and 0.36 respectively.

Calculations of the rarefaction index revealed that the number of pollen taxa (examined for a large but constant number of pollen grains) in samples from non-forest communities and young growths is almost identical. In the former type of communities, the average rarefaction value comes to 10.40 (excluding sample B-M2-N from calculations), whereas in the latter type - 10.29. In the old forests, the rarefaction index is slightly lower and amounts to 9.64 (Table 3).

\subsection{Numerical and statistical analysis of samples described by the composition and the rate of taxa deposition}

High similarity between the surface pollen samples, particularly those coming from non-forest communities and young growths, was confirmed by the numerical classification performed with the software MVSP. Quite often in the dendrogram drawn up with the software MVSP, samples from these plant communities form common clusters - see the upper and lower part of Figure 4. Homogeneous groups, i.e. containing samples collected in one type of vegetation, are seldom distinguished at a low fusion level, corresponding to a high percentage of similarity. These are just a group comprising samples A-T1-N and B-G1-N from the heaths of both studied regions, as well as a threeelement group of samples A-P1-O, A-R1-O and A-T1-O from old forests in the Tuchola Forest. At the fusion levels exceeding $60 \%$ of similarity, they form bigger groups with samples collected in all types of communities, i.e. $\mathrm{N}$, $\mathrm{Y}$ and $\mathrm{O}$.

Also in the ordination diagram drawn with the Principal Component Analysis, the surface pollen samples from different communities are situated close to each other. In the clouds of data points distributed in the system of axes I and II, it is not possible to draw homogeneous subsets comprising only samples $\mathrm{N}, \mathrm{Y}$ or O (Fig. 5).

The first two principal axes explain only $23.36 \%$ and $13.76 \%$ of the variance, respectively. The first principal component axis splits the pollen spectra into two groups. Spectra A-R1,-N A-P1-N and A-L1-Y with the highest abundance of Calluna pollen grains have mostly negative values on the first axis, while samples B-M2-N and B-Z3-O without Calluna pollen grains have positive values. In the latter samples, collected in the suburb of Torun, where besides forests and heaths, a large area is covered with home gardens, quite a lot of pollen grains of Brassicaceae and Chenopodiaceae were recorded.

The second principal component axis differentiates a group of samples collected in old forest of the Tuchola Forest (A-P2-N, A-L2-Y, A-P2-O, A-R1-O), in which quite a lot of Picea pollen grains occurred. The position of this group corresponds to high values on the second axis. In the lower part of the diagram, corresponding to negative values on the second axis, there are mainly pollen spectra from young forests and heaths of the Torun Basin (B-M1Y, B-M1-N, B-G2-N), in which spruce is not recorded.

High similarity of surface pollen samples from different communities is also confirmed by statistical tests. The Kruskal-Wallis test revealed that the differences in pollen rain in three types of the studied phytocoenoses are sta- 
UPGMA

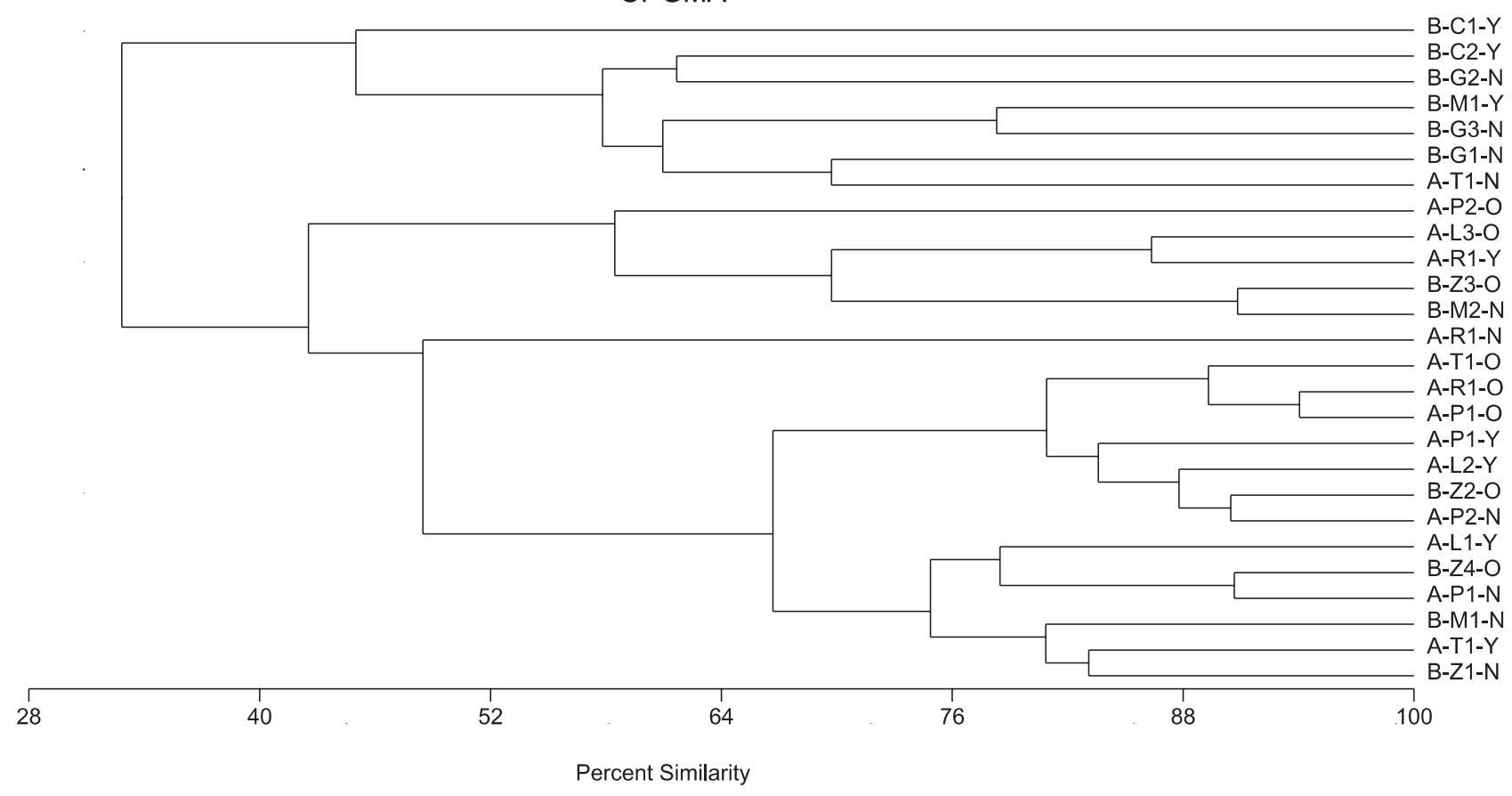

Figure 4. Dendrogram of 26 surface pollen spectra based on the taxa composition and deposition of their pollen (clustered with UPGMA and the percentage similarity index)

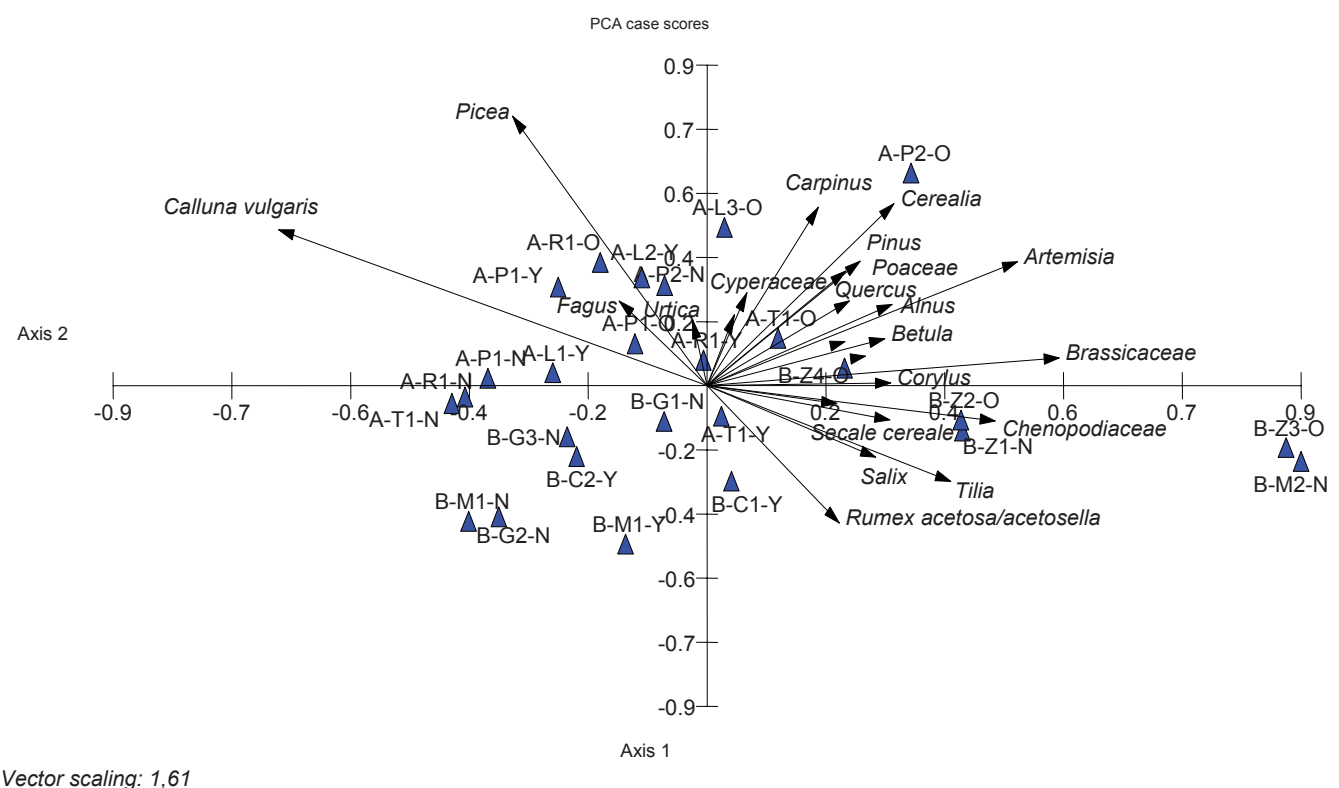

Figure 5. Distribution of pollen samples presented in Figure 3 in the plane of axes 1 and 2, determined by PCA 
tistically significant only in the case of Pinus $(\mathrm{p}=0.020)$, Betula $(\mathrm{p}=0.0077)$ and Quercus $(\mathrm{p}=0.0008)$. Whereas the results of the post-hoc test revealed that the pollen rain of Pinus differed in the communities $\mathrm{O}$ and $\mathrm{N}(\mathrm{p}=0.023)$, the pollen rain of Betula differed also in the communities $\mathrm{O}$ and $\mathrm{N}(\mathrm{p}=0.0056)$, and in the case of Quercus - both in the communities $\mathrm{O}$ and $\mathrm{N}(\mathrm{p}=0.0008)$, as well as in the communities $\mathrm{O}$ and $\mathrm{Y}(\mathrm{p}=0.021)$.

Among herbaceous plants, significant differences were recorded for Poaceae (the Kruskal-Wallis test, $\mathrm{p}=0.015$ ), Brassicaceae $(\mathrm{p}=0.047)$ and Artemisia $(\mathrm{p}=0.0016)$. Whereas the results of the post-hoc test revealed that in the case of the first two taxa, differences in the pollen deposition occurred between the communities $\mathrm{O}$ and $\mathrm{N}$ ( $\mathrm{p}=0.011$ and $\mathrm{p}=0.059$, respectively). Deposition of Artemisia pollen differed significantly both between sites $\mathrm{O}$ and $\mathrm{N}(\mathrm{p}=0.025)$, and between $\mathrm{O}$ and $\mathrm{Y}(\mathrm{p}=0.014)$.

\subsection{The structure of surface samples and their numerical classification based on the percentage contribution of pollen taxa}

In particular surface samples, pollen of Pinus occurred most abundantly. Its contribution usually exceeded 50\% of the total number of pollen grains. Only in two samples from the non-forest communities (A-R1-N and B-G2-N) and two samples from young growths at the forest burnt site in the village of Cierpice (B-C1-Y and B-C2-Y), its contribution was less than $50 \%$ (Fig. 6). The contribution of pine pollen in samples from the non-forest communities varied considerably, between $35.03 \%$ in sample B-G2-N from the heathland in the city district of Glinki and $80.10 \%$ in the heathland B-M1-N under the power line in the village of Mała Nieszawka.

In the surface samples from the non-forest communities, where the contribution of Pinus was small, the pollen of Ericaceae (including Calluna and Arctostaphylos) was the most significant. In sample A-R1-N, the pollen of Pinus constituted $37.34 \%$ and the pollen of Ericaceae $54.19 \%$. In sample B-G2-N, the contribution of pollen of these taxa amounted to 35.03 and $43.24 \%$, respectively. The only samples were pollen of Ericaceae was not recorded was sample B-Z1-N collected in the patch of heather on the edge of the forest covering the Zadroże Dune and sample B-M2-N from the wartime cemetery located next to this dune. The contribution of Betula pollen in samples from the non-forest communities ranged from 2.31 to $11.92 \%$ (in sample B-G2-N), and the contribution of Poaceae pollen - from 0 to $3.10 \%$.

In samples collected in young growths, the contribution of Pinus pollen ranged from $25.80 \%$ in the birch monoculture at the forest burnt site in the village of Cierpice in the Torun Basin, to $88.10 \%$ at site A-R1-Y at the airstrip near the village of Rolbik in the Tuchola Forest. Among all the samples collected in young forest stands, the contribution of Betula pollen was the highest in the former sample, whereas the smallest one - in the latter one.

The contribution of Ericaceae pollen in this type of plant communities ranged from $0.85 \%$ in the young pine wood adjacent to the heathland under the power line in the village of Mała Nieszawka to $21.31 \%$ in sample A-P1-Y collected in the young birch-pine growth at the boundary with the Tuchola Forest National Park. The latter sample had the smallest contribution of Poaceae pollen $(0.84 \%)$ among all the samples from young growths. The highest contribution of Poaceae in the surface samples from this category of communities, i.e. $12.52 \%$, was recorded in sample A-T1-Y from the airstrip in the Forest Inspectorate of Tuchola.

In all the surface samples collected in old forests, the contribution of Pinus pollen exceeded 50\%, and it ranged from $50.39 \%$ in sample B-Z4-O collected on the Zadroże Dune in the Torun Basin to $88.14 \%$ in sample A-L3-O from the Tuchola Forest National Park. The latter value was the highest contribution of Pinus in the entire set of 26 surface pollen samples.

The contribution of Betula pollen in the subset of samples from old forests ranged from $3.39 \%$ in the sample from fresh coniferous forest in the Tuchola Forest National Park to $16.70 \%$ in sample A-T1-O collected in the mature forest at the airstrip in the Forest Division of Tuchola. Whereas, the pollen of Ericaceae constituted between $0.13 \%$ in sample B-Z3-O and 22.07 in sample B-Z4-O. Both samples come from the Zadroże Dune, however the former one was collected in the middle of the forest complex, and the latter one - on the forest edge, where a community with a close affinity to dry pine forest develops.

The smallest and the highest contribution of Poaceae pollen were recorded at the sites in the Tuchola Forest. The smallest contribution of this taxon, just $0.51 \%$, was recorded in sample A-L3-O from the edge of the National Park, and the highest, amounting to $4.86 \%$, in the old forest in the habitat of fresh coniferous forest at the airstrip near the village of Rolbik.

The contribution of other pollen taxa, apart from Pinus, Betula, Ericaceae and Poaceae, was insignificant in the examined samples. The maximum contribution of other AP in samples from non-forest communities, young growths and old forests, amounted to $12.73,17.54$ and $9.41 \%$, respectively. Whereas, the maximum contribution values for other NAP in this series of samples were 6.22, 4.46 and $6.05 \%$, respectively.

The percentage contributions of particular pollen types in samples N, Y and $\mathrm{O}$ from the same site (Fig. 7) are very different. In the series of communities at the airstrip near the village of Rolbik (the Forest Inspectorate of Przymuszewo), the spectra of the sample from the heath and the samples from (young and old) forests differed considerably 


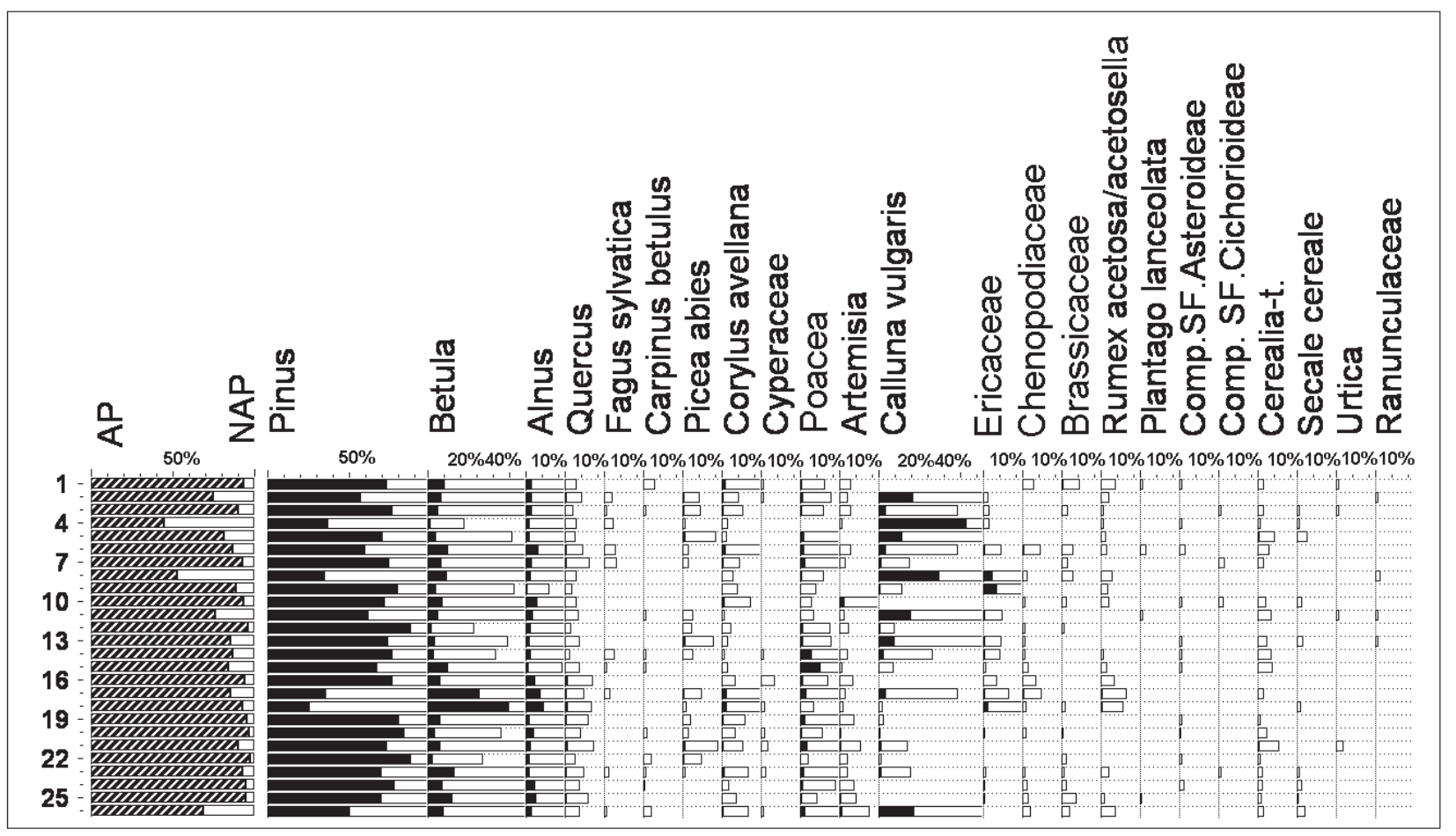

Figure 6. Contribution of pollen taxa expressed as percentage of the total number of pollen grains in pollen spectra of 26 surface samples. Samples are plotted according to vegetation types. Remarks about samples as in Figure 3

in the contribution of Ericaceae and Pinus. The highest (among all the examined samples) contribution of heather $(54.18 \%)$ was recorded in the sample from the non-forest community, together with one of the lowest contribution of pine $(37.34 \%)$. The forest communities of this site were characterized by a considerable contribution of Pinus pollen $(88.10 \%$ in the sample from the young growth and $73.0 \%$ in the sample from the old forest), as well as by small contribution of Ericaceae pollen $(1.03 \%$ in the young stand and $1.78 \%$ in the old forest).

Forest communities from the airstrip near the village of Rolbik differ quite clearly regarding the contribution of pollen of Betula and Poaceae, as well as the remaining AP and NAP. The contribution of pollen of these taxa in the sample from the old forest is $2-2.5$ times higher than in the sample from the young forest (Fig. 7). However, the total pollen count in the sample from the old forest is much lower than in the sample from the young growth, 9,615 and 6,028 grains $/ \mathrm{cm}^{2}$, respectively.

From the diagrams presented in Figure 7, it appears that in the samples collected in the heath, in the young stand and in the old forest at the airstrip in the Forest Inspectorate of Tuchola, the percentage of pine pollen is relatively simi$\operatorname{lar}(70.36,67.16$ and $69.63 \%$, respectively). And thus, the pattern of similarities and differences between the samples differ from those at the site near the village of Rolbik. The contribution of Betula pollen in the sample from the young growth (12.33\%) and from the old forest (16.70\%) near the airfield in the Forest Inspectorate of Tuchola is higher than in the sample from the heath $(5.19 \%)$, ca. two and three times, respectively. The contribution of Poaceae pollen is significantly higher in the sample from the heath than in the sample from the young growth and old forest (14.48, 1.14 and $2.19 \%$, respectively). The contribution of Poaceae pollen in the sample from the young growth $(12.52 \%)$ is much higher than in the samples from the heath $(2.41 \%)$ and old forest $(2.53 \%)$.

The average contribution of Pinus pollen in the groups of samples from the non-forest communities and young growths amounts to 62.16 and $63.22 \%$, respectively. The arithmetic mean for old forests is higher and amounts to $74.27 \%$ (Fig 8). In terms of Betula pollen contribution, quite similar values were obtained for non-forest communities (7.94\%) and old forests (9.38\%). The average contribution of Betula pollen for young growths is higher $(15.11 \%)$. The average contribution of Ericaceae pollen in samples collected in non-forest communities $(18.29 \%)$ was higher than the average value for young growths $(6.31 \%)$, and particularly for old forests $(3.41 \%)$. Whereas, the average contributions of pollen types - other AP and NAP 
A-R1-N

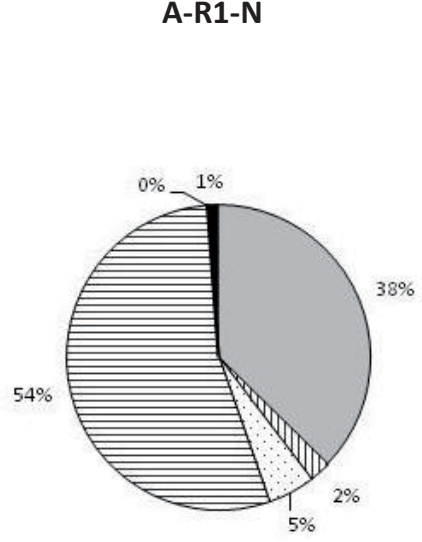

A-T1-N

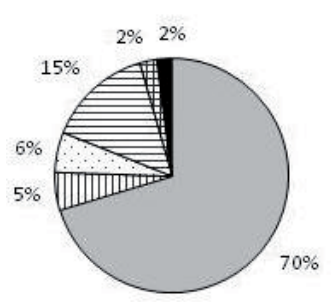

A-R1-Y

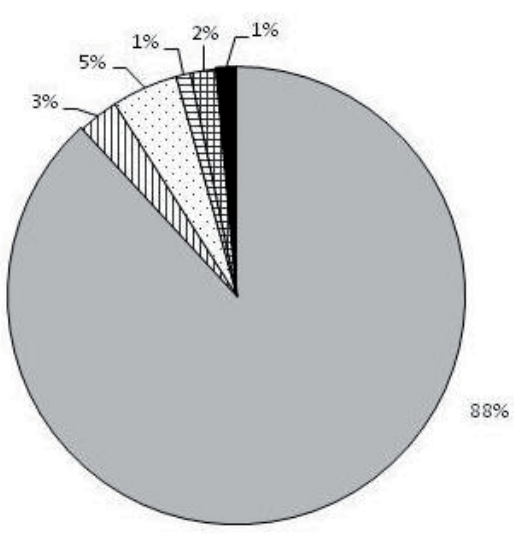

A-T1-Y

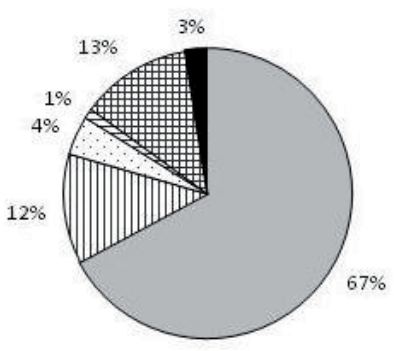

A-R1-0

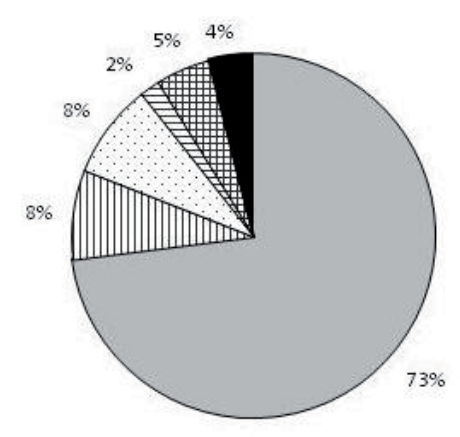

A-T1-0

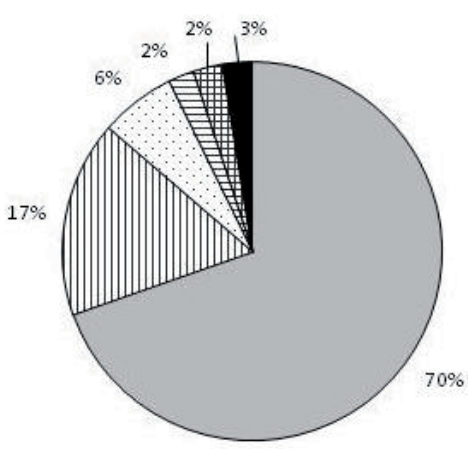

$\square$ Pinus $\mathbb{m}$ Betula $\square$ others AP 目Ericaceae 四Poaceae others NAP

Figure 7. Comparison of deposition and percentage of six main groups of pollen types in samples collected in non-forest communities $(\mathrm{N})$, young growths $(\mathrm{Y})$ and old forests $(\mathrm{O})$ at the airstrip near the village of Rolbik $(\mathrm{R})$ and the airstrip in the Forest Division of Tuchola (T). The size of circles presenting the spectra is proportional to pollen abundance in sample A-T1-N with the lowest number of pollen grains

for the groups of surface samples from non-forest communities, young growths and old forests are quite similar (Fig. 8). In the case of other AP, the arithmetic means amount to $7.20,8.81$ and $7.49 \%$, respectively. For other NAP, the arithmetic means have values of $2.71,2.70$ and $3.10 \%$, respectively.

Sample B-M2-N, not included in these comparisons, from the wartime cemetery in the village of Mała Nieszaw$\mathrm{ka}$, differed from the average values in the absence of Ericaceae pollen, low contribution of Poaceae pollen $(0.71 \%)$, as well as higher contribution of other AP (12.0\%) and other NAP $(6.11 \%)$. The contribution of Pinus pollen in this sample reached the value of $71.68 \%$ and was most similar to the average contribution value for this type of pollen in the samples from old forests $(74.27 \%)$.
The Kruskal-Wallis test, carried out for the set of 25 samples (excluding the missing sample B-M2-N), revealed that the differences in the structure of pollen deposition in three types of the studied phytocoenoses are statistically significant only in the case of Ericaceae $(p=0.042)$. The results of the post-hoc test indicated, however, that the pollen deposition of this taxon differed in the communities $\mathrm{O}$ and $\mathrm{N}(\mathrm{p}=0.036)$.

The lack of distinct differences between the pollen spectra of surface samples, collected in three types of communities was confirmed by numerical classification. In the dendrogram drawn on the basis of the percentage composition of taxa at the similarity level of $60 \%$, only three clusters are distinguished, which comprise samples collected in all three types of communities. Small but homogene- 


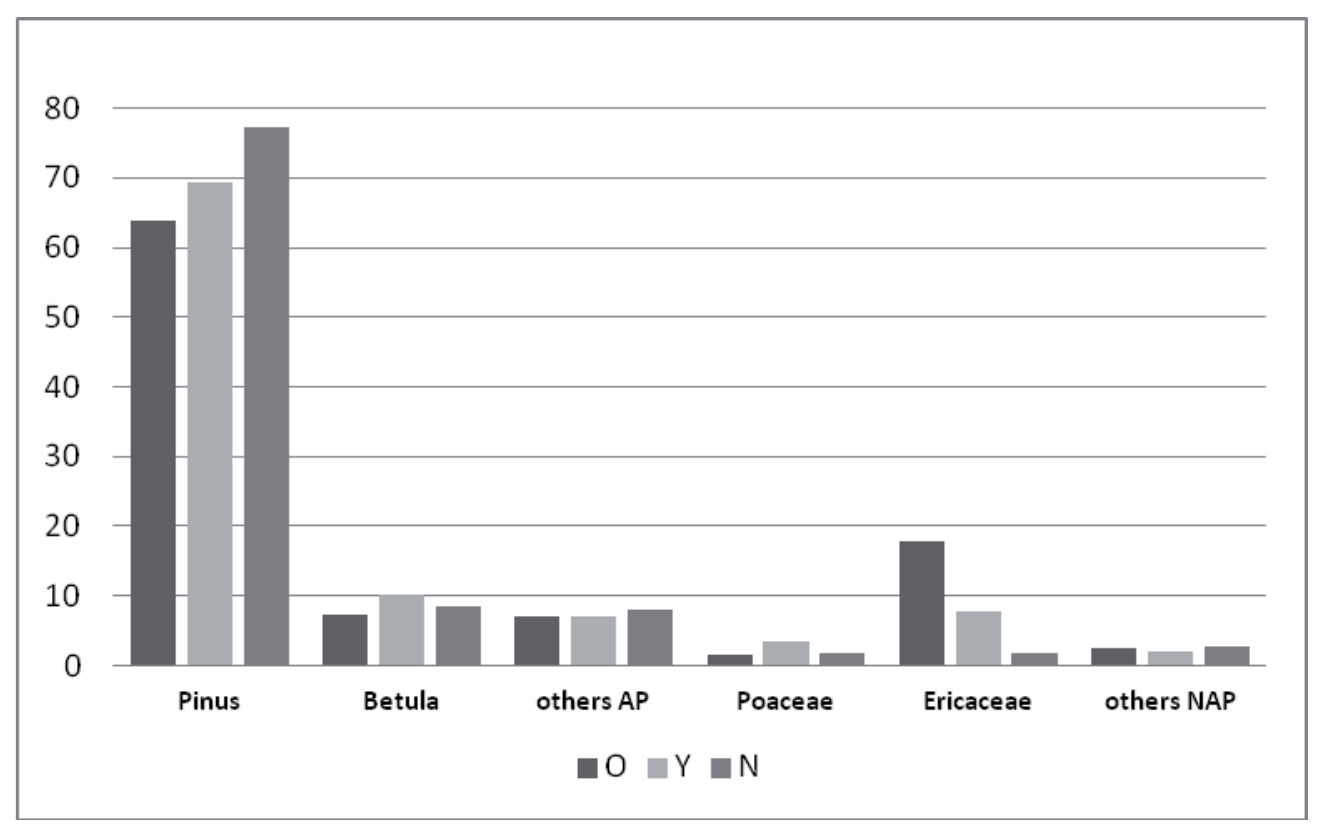

Figure 8. The comparison of average percentage contribution of six main groups of pollen taxa in three types of phytocoenoses (a pollen sample from the wartime cemetery was omitted in the analysis). Explanation of abbreviations as in Figure 7

ous clusters of samples collected within the same type of communities are distinguished at the fusion levels corresponding to the percentage similarity much higher than $60 \%$. Most often these are clusters comprising the surface samples collected in non-forest communities. In the dendrogram there are three two-element clusters of such samples (Fig. 9, clusters: A-L1-N and A-T1-N, A-R1-N and B-G2-N, as well as B-M2-N and B-Z1-N). Moreover, one two-element cluster of samples collected in young stands of the Torun Basin was clearly distinguished (samples B$\mathrm{C} 1-\mathrm{Y}$ and $\mathrm{B}-\mathrm{C} 2-\mathrm{Y}$ ) and one two-element cluster of surface samples from old forests (A-T1-O and B-Z3-O). Nevertheless, as in the case of classification based on the density of pollen of particular taxa, also in this dendrogram, homogeneous clusters group together at the levels corresponding to a high percentage of similarity with the surface samples collected in other types of communities (Fig. 9).

A high similarity between pollen samples from three different types of communities is also apparent on the PCA biplot. Points representing samples from non-forest communities, as well as samples from young and old forests are often located close to each other (Fig. 10). In the case of analysis carried out on samples characterized by the percentage contribution of pollen taxa, the first two principal component axes explain a higher per cent of the variability, as compared to the analysis carried out on samples characterized by the absolute density of pollen from particular taxa.
In the analysis, the results of which is presented in Figure 10 , the first axis accounts for $44.46 \%$ and the second axis - for $17.84 \%$ of the variation. In this analysis, the first axis separates pollen samples with the highest contribution of Calluna (A-R1-N, A-P1-N, A-P1-Y) from samples without pollen of this taxon (B-Z1-O, B-Z2-O, B-Z3-O, B-M2-N). The former ones, mainly samples collected from large heathlands, are situated on the right side of the diagram corresponding to positive values along the first axis. The latter ones, collected in old forests, are distributed at the left edge of the diagram, corresponding to the lowest, negative values along the first axis.

Whereas the second axis separates pollen spectra with high contribution of Betula (B-C1-Y, B-C2-Y), which are located in the upper left quadrant of the diagram corresponding to negative values on the first axis and positive values on the second axis, from pollen spectra with high contribution of Pinus pollen (A-L3-O, A-R1-Y), which are situated in the lower part of the diagram with negative values on the second axis.

\subsection{Evaluation of the relationship between the vegetation structure and the pollen spectrum}

When determining the influence of vegetation on the pollen spectrum, one should compare both the species composition and the quantitative relationships between components that occur within a patch of vegetation, as well as in a pol- 
UPGMA

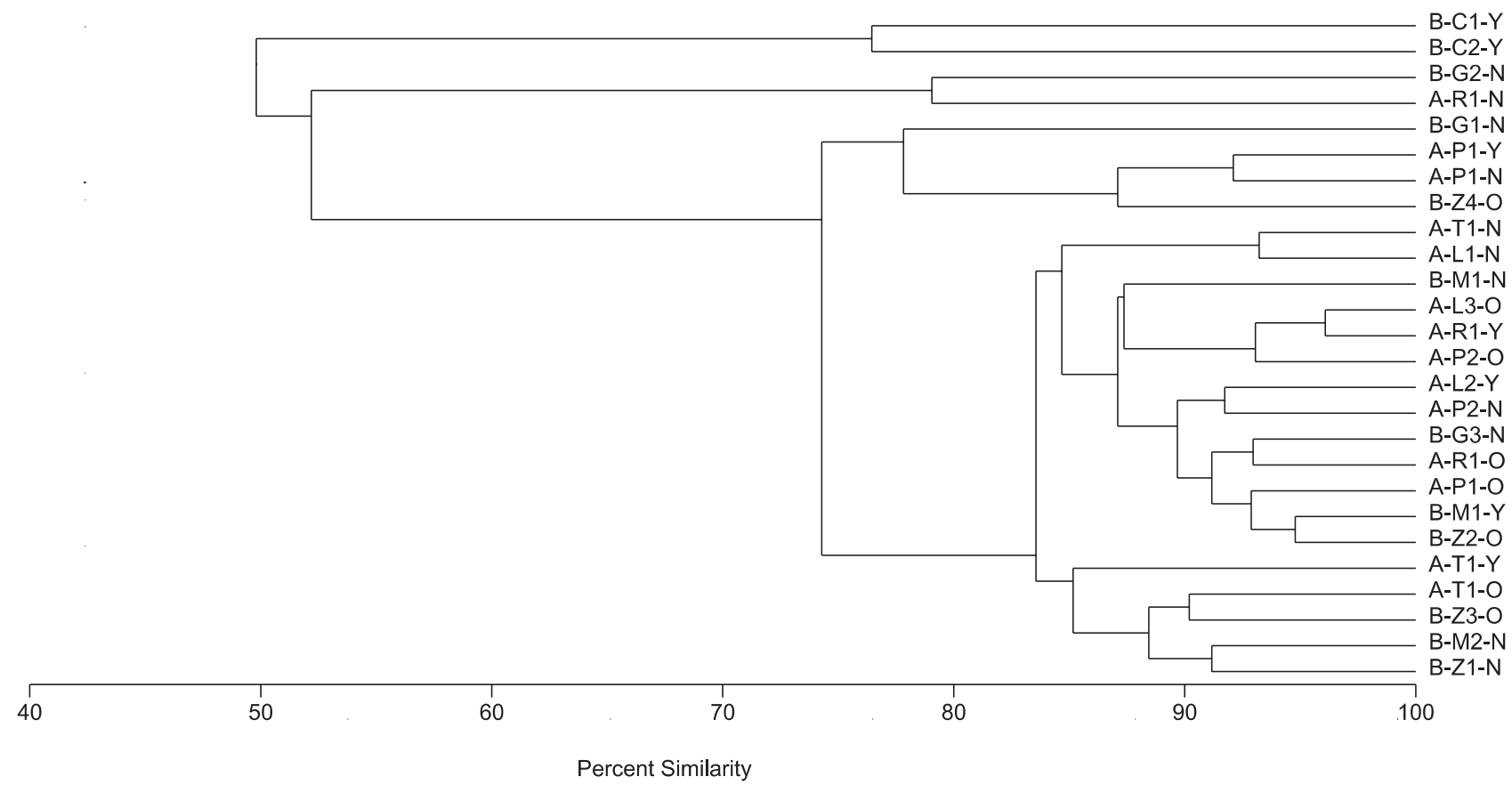

Figure 9. Dendrogram of 26 surface pollen samples based on the pollen taxa composition and their relative percentage contribution (clustered with UPGMA and the percentage similarity index). Explanation of abbreviations as in Figure 7

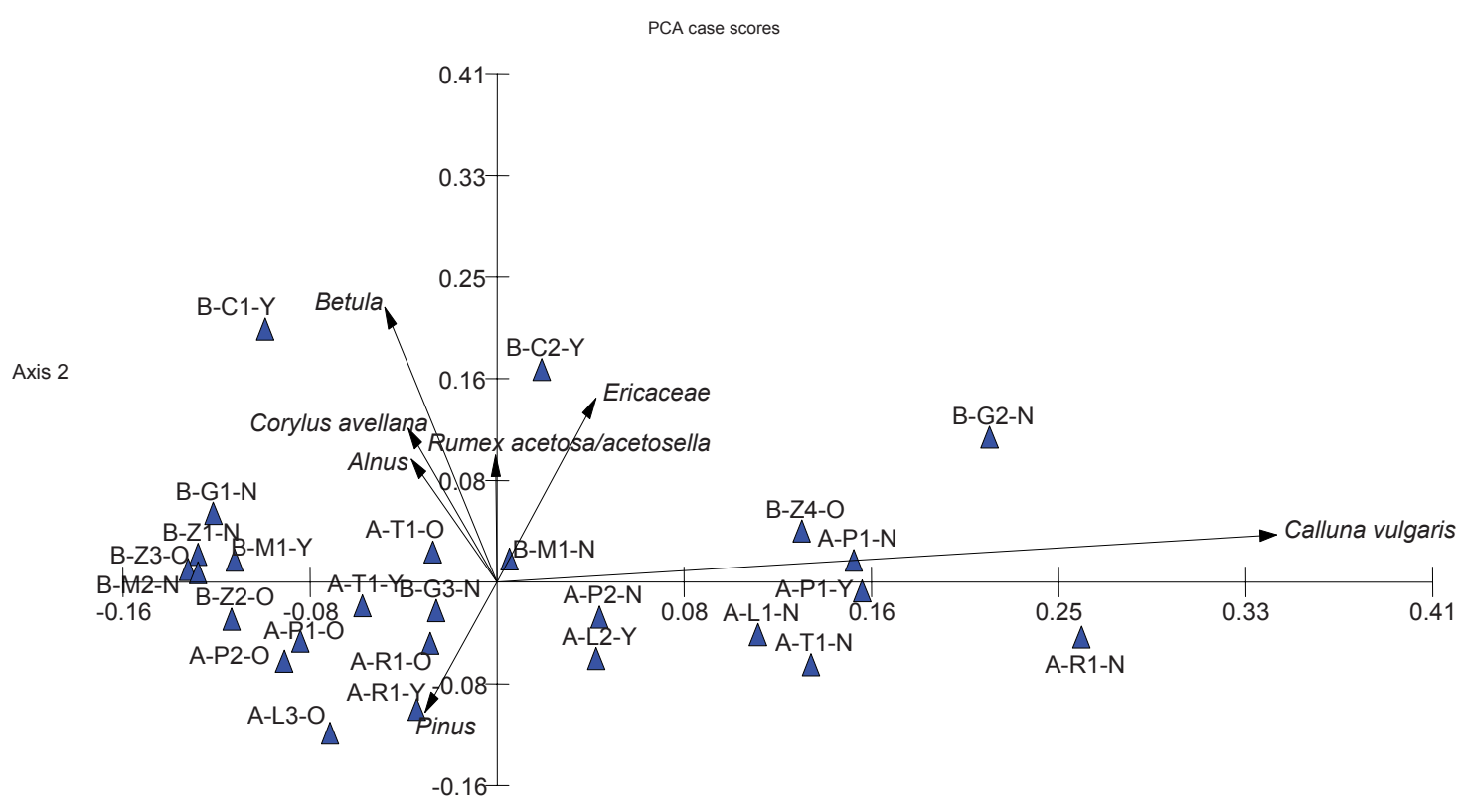

Vector scaling: 0,36

Axis 1

Figure 10. Distribution of pollen samples presented in Figure 6 in the plane of axes 1 and 2, determined by PCA 

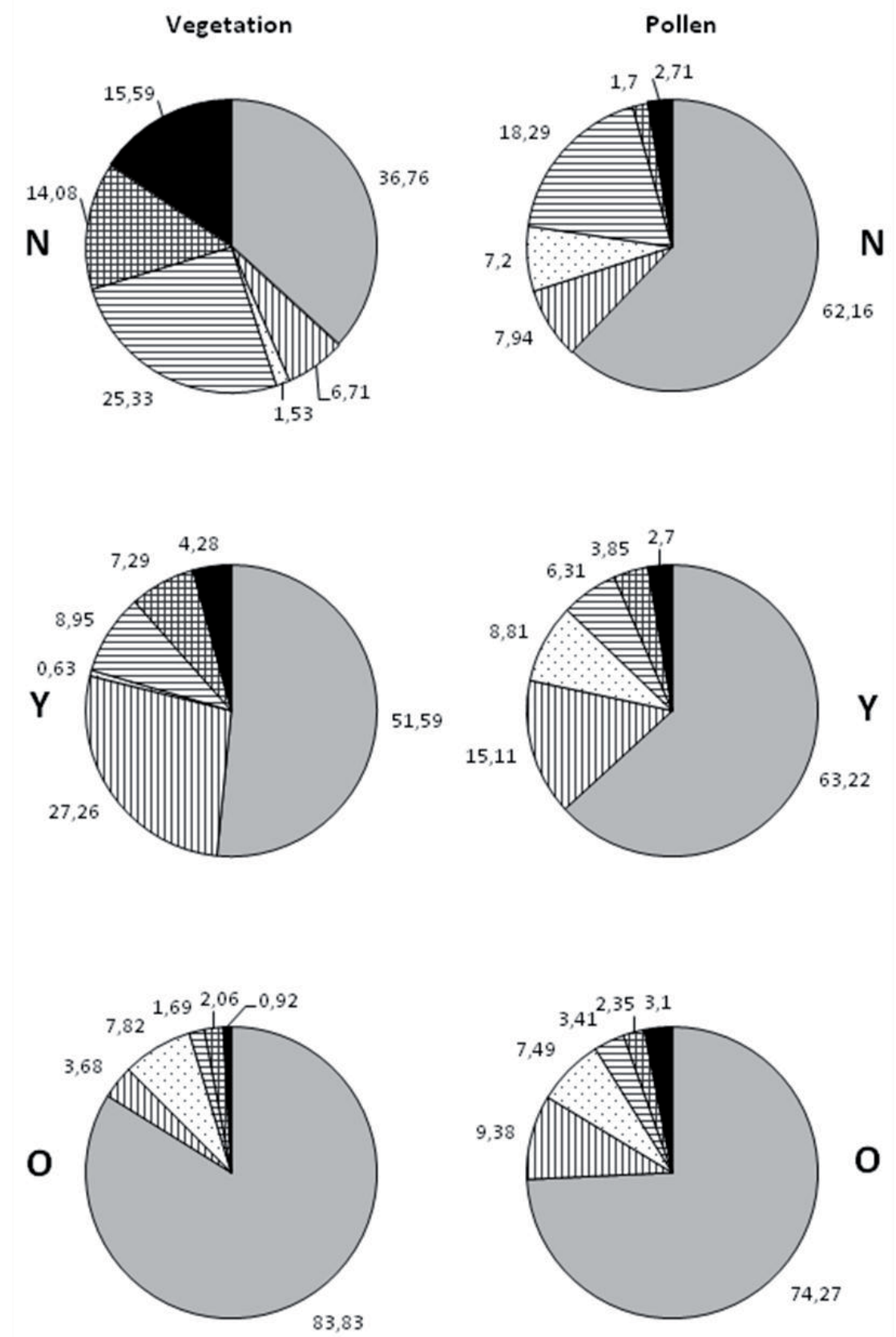

\section{$\square$ Pinus $\mathbf{m}$ Betula $\square$ others AP 目Ericaceae 曲Poaceae $\square$ others NAP}

Figure 11. Comparison of the vegetation structure and pollen spectra for three types of phytocoenoses. Values are presented as average percentage from nine or eight sites, both for vegetation and surface samples (a pollen sample and a relevé from the wartime cemetery were omitted in the analysis). Vegetation percentage values are presented as the proportion of the total vegetation abundance per relevé 
len sample collected within that patch. Average values for the contribution of species and groups of species in three types of plant communities, as well as pollen types corresponding to these species in three groups of surface samples are presented by diagrams in Figure 11. When comparing the corresponding samples from each of the three pairs of diagrams, it appears that quantitative relationships between taxa in relevés and pollen samples develop quite differently. The differences are related mainly to a smaller percentage of Pinus in relevés from non-forest communities and young growths, as well as higher contribution of this component in old forests, as compared to relevant values in groups of pollen samples. Despite the application of conversion factors, which express the relative differences in the abundance of species from the layers $\mathrm{a}_{1}, \mathrm{a}_{2}, \mathrm{~b}$ and $\mathrm{c}$ in the studied phytocoenoses, the pine domination in the structure of old forests is not as high as its domination in the pollen spectra.

Other major discrepancies occur with higher contribution of taxa of Ericaceae, Poaceae and other NAP in relevés from non-forest communities in relation to contribution of these components in a relevant group of pollen samples. Also the percentage contribution of birch in relevés from young growths is much higher than the contribution of birch pollen in surface samples from these communities. Furthermore, the contribution of the category defined as other AP in relevés from non-forest communities and young growths is much lower as compared to corresponding pollen taxa in the corresponding groups of the surface samples.

The association size is a good indicator of the relationship between components of a phytocoenosis and pollen taxa of surface samples collected in those phytocoenoses. Based on the results of the analysis of the set of 25 relevés and the corresponding set of 25 pollen samples, it was found that only Calluna vulgaris is a strongly associated pollen type. The index describing the relations between the occurrence of this species in the vegetation and in the pollen samples reached the value of 0.73 . While the following taxa should be classified as associated types: Galium, Poaceae, Compositae SF. Asteroideae, Pinus, Compositae SF. Cichorioideae, Arctostaphylos, Rumex acetosa/ acetosella and Betula. Ericaceae and Chenopodiaceae were classified as weakly associated types. The complete list of associated and unassociated types of taxa, including the overrepresented and underrepresented ones is included in Table 4.

\section{Discussion and conclusions}

The performed analyses revealed quite big differences between the pollen samples collected in the same types of plant communities. At the same time, it was observed that samples from different types of communities formed common, rather small in number, clusters. On the one hand, these similarities and differences prove the heterogeneity of samples coming from phytocoenoses of the same type, which was confirmed by statistical analyses, and on the other hand, they indicate a high similarity between samples coming from different types of communities.

High variability of pollen samples collected in the same type of phytocoenoses, e.g. young stands, was affected by the fact that they were collected both in pine or birch monocultures, as well as in plantations of both species. Older pine forest stands, however, especially in the Tuchola Forest region, occurred in different habitats - both in the habitat of dry and fresh coniferous forests. On the other hand, in older forest stands from the Torun Basin, the herb layer was very poor in species. In the pine forest stand on the Zadroże Dune, which constitutes the first generation of trees on the former heathland and psammophilous grassland, usually only pine needles were lying on the forest floor and no plant species occurred.

Also heaths, where surface pollen samples were collected, were characterized by high structural variability. At particular non-forest sites, the vegetation mosaic was composed of patches of Calluna vulgaris and psammophilous grasslands of different sizes.

The abundance and taxonomic composition of pollen in surface samples is probably also affected by characteristics of the substratum at which pollen is deposited. Mulder (1997) reported that pollen grains are basically well preserved in the moss cover, therefore in samples collected from this type of substratum, one can identify many types of pollen grains, and samples collected from mosses can be used in modern pollen rain analysis.

In the analysis described in this paper, different moss species occurred in the areas from which pollen samples were collected. In the case of young growths and old forests, those were mainly Pleurozium schreberi and Dicranum undulatum. In the case of non-forest communities, apart from both aforementioned species, it was Polytrichum piliferum and sometimes also lichens, which occurred among mosses. Besides differences in the species composition of mosses and lichens, the qualitative and quantitative characteristics of a pollen sample could be influenced by different water saturation of the vegetation in the herb layer at different sites. In the forest communities, it was usually quite high during all the seasons. In the nonforest communities, with a considerable inflow of direct solar radiation, particularly during the summer months, twigs of mosses and thalli of lichens are sometimes very dry. In the analysed set of samples, differences in the local climatic conditions occurring in the Tuchola Forest and in the Torun Basin could enhance the variability resulting from the influence of different water saturation of plants. In the former area, the presence of numerous lakes and rivers 
Table 4. Indices of associations (A), under-representation (U) and over-representation $(\mathrm{O})$ for taxa present in both data sets, i.e. vegetation and pollen spectra

\begin{tabular}{|c|c|c|c|}
\hline Pollen type & A & $\mathrm{U}$ & $\mathrm{O}$ \\
\hline \multicolumn{4}{|c|}{ Strongly associated types } \\
\hline Calluna & 0.73 & 0 & 0.27 \\
\hline \multicolumn{4}{|c|}{ Associated types } \\
\hline Galium & 0.33 & 0.50 & 0.50 \\
\hline Poaceae & 0.32 & 0.51 & 0.52 \\
\hline Compositae SF. Asteroideae & 0.22 & 0.60 & 0.67 \\
\hline Pinus & 0.21 & 0.58 & 0.70 \\
\hline Compositae SF. Cichorioidae & 0.20 & 0.67 & 0.67 \\
\hline Arctostaphylos & 0.17 & 0.80 & 0.50 \\
\hline Rumex acetosa/acetosella & 0.15 & 0.56 & 0.81 \\
\hline Betula & 0.12 & 0.77 & 0.81 \\
\hline \multicolumn{4}{|c|}{ Weakly associated types } \\
\hline Ericaceae & 0.08 & 0.71 & 0.89 \\
\hline Chenopodiaceae & 0.06 & 0.50 & 0.94 \\
\hline \multicolumn{4}{|c|}{ Unassociated types } \\
\hline Artemisia & 0 & 1.00 & 1.00 \\
\hline Fabaceae & 0 & 1.00 & 1.00 \\
\hline Caryophyllaceae & 0 & 1.00 & 1.00 \\
\hline Cyperaceae & 0 & 1.00 & 1.00 \\
\hline Rosaceae & 0 & 1.00 & 1.00 \\
\hline Plantago major/media $\mathrm{t}$. & 0 & 0 & 1.00 \\
\hline Polygonum & 0 & 0 & 1.00 \\
\hline Polypodiaceae & 0 & 0 & 1.00 \\
\hline Ranunculaceae & 0 & 0 & 1.00 \\
\hline Urtica & 0 & 0 & 1.00 \\
\hline Apiaceae & 0 & 1.00 & 0 \\
\hline Campanulaceae & 0 & 1.00 & 0 \\
\hline Euphorbia & 0 & 1.00 & 0 \\
\hline Juncaceae & 0 & 1.00 & 0 \\
\hline Lamiaceae & 0 & 1.00 & 0 \\
\hline
\end{tabular}

increases the air humidity. On the outskirts of the city of Torun and in the areas bordering on the city, particularly in places more distant from the Vistula River, the air humidity is probably lower.

When evaluating the influence of the substratum on the abundance and the pollen species composition in surface samples, the Tauber traps (1974) are worth applying as a reference point, as it was done by Fontana $(2003,2005)$ in her studies on the relationship between the vegetation and the modern pollen rain on coastal dunes within the province of Buenos Aires in Argentina.

On the other hand, high similarity between pollen samples collected from different plant communities, as it was revealed in the analyses, results mainly from two reasons. Considerable domination of pine pollen in pollen spectra is the first reason. It is particularly evident in areas where pine forests dominate in the landscape, such as Tuchola Forest and Torun Basin. The easiness with which pine pollen spreads over considerable distances and its intensive inflow from outside a phytocoenosis, i.e. from a landscape where pine forests dominate, cause that differences between samples get reduced, including samples from forest and non-forest plant communities.

Indeed, the Scots pine pollen had the highest contribution in the studied set of 26 samples among other species of trees and shrubs. It occurs in all pollen spectra in big quantities regardless of the habitat type. These observations are in line with the results of analyses described by other researchers. Among others, Belgrund et al. (1986) and Mulder (1997) reported that Scots pine occurs in big quantities, not only in samples coming from coniferous forests, but also in non-forest samples.

Not only the presence of a considerable amount of Scots pine pollen in all samples reduces the differences between pollen samples collected in different types of phytocoenoses, but as it was pointed out by Makohonienko et al. (1993) - a similar effect is also exerted by a number of other taxa, e.g. Poaceae. Pollen of this taxon is frequently encountered in big quantities regardless of a plant community in which a given pollen sample was collected. The following taxa are listed by the authors among other pollen taxa that can be observed both in forest and non-forest samples: Artemisia, Urtica, Chenopodiaceae, Rumex acetosa/acetosella., Cerealia-t. Furthermore, Makohonienko (1993) pointed out the fact that palynological data from afforested areas, including areas covered with pine forests, as well as data from meadow areas, can be clearly separated in numerical analyses.

Our research revealed that samples collected at afforested sites are characterized by higher percentage content of tree pollen as compared to samples coming from unforested areas. Also Makohonienko et al. (1993) reported that pollen of trees occur in larger quantities in afforested areas as compared to meadow areas. 
In the analyses of pollen rain samples performed in the present study, the pollen spectra from heaths, young growths and old forests frequently group together, both in agglomerative hierarchical classification and in ordination diagrams (PCA), which proves that the similarity between them is relatively high. Small differences result from high contribution of common species, which has been reported when comparing heaths and non-forest communities, as well as young growths and old forests.

In this paper, no distinct relation was recorded between the structure of a phytocoenosis and a pollen spectrum. The absence of such relationships is proved by the fact that only one species - Calluna vulgaris - demonstrated a strong association between the occurrence in a plant community and in a pollen sample. Also, there were only few species (just 6) with weak association. In the case of open communities growing on coastal dunes in Argentina, Fontana (2005) reported the presence of 3 species with strong association, 3 species with average and 4 species with weak association. The considerable differences observed in our study, between the structure of a phytocoenosis and a pollen spectrum contradict the statement of Kvavadze (1996) that the taxonomic composition of palynological samples is, in most cases, consistent with species composition observed in relevés made at a pollen sample collection site.

In the results obtained from the comparison of 26 palynological samples and relevés from the Tuchola Forest, the high difference between the number of species recorded in relevés and the number of taxa in pollen samples is particularly noteworthy. Despite the fact that pollen samples can often comprise a group of species, the number of taxa recorded in them was almost always higher than in a corresponding relevé. This is caused by the aforementioned possibility of long-distance spreading of pollen grains owing to air movement.

The observations made in our study are in line, however, with the results presented in the study by Gaillard et al. (1994), where it was also found that more taxa occur in pollen samples than in relevés. Also these authors reported that the similarity was caused by distant transportation of pollen from neighbouring and further habitats, as well as from plant communities.

Gaillard et al. (1994) also observed that numerical analyses reveal considerable differences between pollen spectra from afforested and grazed meadow areas, and thus nonforest communities. This conclusion is in contradiction with the results of our numerical analyses, which indicate that differences between non-forest and forest communities are minor. This kind of relationship, however, concerns the comparisons of heaths and pine forests, and not deciduous forests and grazing lands of good quality.

Bigger differences between a phytocoenosis and a pollen spectrum for the forest sites than for the open plant communities constitute the next conclusion resulting from the present paper. Perhaps the canopy of tree stands enhances these differences, by reducing the inflow of pollen grains from the surroundings. High frequency of overproductive pollen taxa usually reduces the differences between samples.

It also appears from our observations that the larger the area covered by heaths in the landscape, the greater the differences between pollen spectra collected from heaths and forests. This was particularly evident in the subset of samples collected at the airstrip near the village of Rolbik. As compared to all non-forest sites, the heath covered the largest area here. Also the Calluna pollen constitutes the largest fraction, both as regards the density and the percentage contribution. On the other hand, in other samples from the Tuchola Forest, as well as in samples from the Torun Basin, if the heath area was small, the quantitative and qualitative contribution of heather pollen in samples was insignificant. Also, the similarity of surface pollen spectra from non-forest and forest areas was higher.

Based on the above observations, one can formulate the main conclusion resulting from the present study, i.e. a pollen spectrum is more influenced by the landscape structure than by the structure of a phytocoenosis. In order to check this thesis, it is necessary, however, to carry out studies on the representativeness of a pollen sample, which consist in defining the size and the vegetation cover of the area from which the main part of pollen grains in this sample comes from. In this kind of analysis, methods proposed by e.g. Sugita (1994) and Sugita et al. (1999) should be applied.

The statement of Gaillard et al. (1992) saying that the analysis of modern pollen spectra can be helpful in the interpretation of fossil pollen diagrams constitutes an important conclusion underlining the usefulness of our analyses. Attempts at using the modern pollen spectra were undertaken by e.g. Broström et al. $(1998,2004)$ and Court-Picon et al. (2005). Taking into account the standpoint of Gillard et al. (1992), in our previous study on the analysis of modern pollen rain in the Tuchola Forest (Hrynowiecka-Czmielewska et al. 2007), we wrote that in order to perform such an interpretation, one should carry out analysis of four data sets: the land use structure/the land cover, relevés, surface pollen samples, if possible collected from the same substratum and with the use of Tauber traps, as well as superstrata of bottom sediments in lakes situated in the vicinity of locations where surface samples were collected. More samples should be collected for this kind of studies, which would make a comparison more objective. Such a complete data set would allow a complete comparison of analysed plant communities, and consequently a more reliable interpretation of fossil pollen diagrams. The final conclusion resulting from our previous researches, as well as from the results obtained in the present study, is the statement that it is worth carrying out this kind of comparison. 


\section{Acknowledgements}

The study was conducted partially within the framework of the grant by the State Committee for Scientific Research No. 3P06L 00825 for the years 2003-2006 and grants by the Ministry of Science and Higher Education No. N N304 220835 and No. N N305 336834.

The authors are grateful to employees of the Tuchola Forest National Park, as well as the Forest Inspectorates of Przymuszewo and Cierpiszewo from the Regional Directorate of State Forests in the city of Torun for making the inventory data on the research sites available.

\section{References}

Andersen S. T., 1970, The relative pollen productivity and pollen representation of North European trees, and corrections factors for tree pollen spectra, Danmarks Geologiske Undersogelse (Series II) 96: 1-99.

Berglund B. E., Emanuelsson U., Persson S. \& Persson T., 1986, Pollen-vegetation relationships in grazed and moved plant communities of south Sweden, [in:] K.-E. Behre (ed.), Anthropogenic indicators in pollen diagrams, A. A. Balkema, Rotterdam: 37-51.

Berglund B. E. \& Ralska-Jasiewiczowa M., 1986, Pollen analysis and pollen diagrams, [in:] B. E. Berglund (ed.) Handbook of Holocene Palaeoecology and Palaeohydrology, John Wiley \& Sons Ltd., Chichester: 455-479.

Birks H. J. B., Line J. M. \& Persson T., 1988, Quantitative estimation of human impact on cultural landscape development, [in:] H. H. Birks, H. J. B. Birks, P. E. Kaland, D. Moe (eds.), The cultural landscape past, present and future, Cambridge University Press, Cambridge: 229-240.

Bradshaw R. H. W., 1981, Modern pollen-representation factors for woods in south-east England, Journal of Ecology 69: 45-70.

Bradshaw R. H. W. \& Webb T. III, 1985, Relationship between contemporary pollen and vegetation data from Wisconsin and Michigan, USA, Ecology 66: 721-737.

Brem M. \& Sobolewska M., 1939, studia nad opadem pyłku drzew leśnych w Puszczy Białowieskiej [The studdus of tree pollen rain in Białowieża Primeval], Sylvan, Ser. A (3-4): 1-10.

Broström A., Gaillard M. J., Ihse M. \& Odgaard B., 1998, Pollen landscape relationships in modern analogues of ancient cultural landscapes in southern Sweden - a first step towards quantification of vegetation openness in the past, Veg. Hist. Archaeobot. 7: 189-201.

Broström A., Sugita S. \& Gaillard M.-J., 2004, Pollen productivity estimates for reconstruction of past vegetation cover in the cultural landscape of Southern Sweden, The Holocene 14: 371-384.

Bunting M. J., 2002, Detecting woodland remnants in cultural landscapes: modern pollen deposition around small woodlands in north-west Scotland, The Holocene 12 (3): 291-301.

Bunting M. J. \& Hjelle K. L., 2010, Effect of vegetation data collection strategies on estimates of relevant source area of pollen (RSAP) and relative pollen productivity estimates (relative PPE) for non-arboreal taxa, Veget. Hist. Archaeobot. 19: 365-374.

Calcote R., 1995, Pollen source area and pollen productivity: evidence from forest hollows, Journal of Ecology 83: 591-602.

Chojnacka J., Cyzman W., Nienartowicz A. \& Deptuła M., 2010a, Variability of the structure and directions of the development of heaths and psammophilous grasslands within the artillery range near the city of Torun, Ecological Questions 12: 87-125.

Chojnacka J., Cyzman W., Nienartowicz A. \& Deptuła M., 2010b, Comparison of heath communities from the class Nardo-Callunetea from the Torun Basin and other regions of Poland, Ecological Questions 12: 127-144.

Court-Picon M., Buttler A. \& Beaulieu J.-L., 2005, Modern pollen-vegetation relationships in the Champsaur valley (French Alps) and their potential in the interpretation of fossil pollen records of past cultural landscapes, Review of Palaeobotany and Palynology 135: 13-39.

Davis M. B., 1963, On the theory of pollen analysis, American Journal of Science 261: 897-912.

Davis O. K., 1984, Pollen frequencies reflect vegetation patterns in Great Basin (U.S.A.) mountains range, Review of Palaeobotany and Palynology 40: 295-315.

Davis R. D. \& Webb T. III, 1975, The contemporary distribution of pollen in eastern North America: a comparison with the vegetation, Quaternary Research 5: 395-434.

Dąbrowski M., 1975, Tree pollen rain and the vegetation of the Białowieża National Park, Biuletyn Geologiczny 19: 157-172.

Fontana S. L., 2003, Pollen deposition in coastal dunes, south Buenos Aires Province, Argentina. Review of Palaeobotany and Palynology 126: 17-37.

Fontana S. L., 2005, Coastal dune vegetation and pollen representation in south Buenos Aires Province, Argentina, J. Biogeogr. 32: 719-735.

Gaillard M. J., Birks H. J. B., Emanuelsson U. \& Berglund B. E., 1992, Modern pollen/land-use relationships as an aid in the reconstruction of past land uses and cultural landscapes: an example from south Sweden, Veg. Hist. Archaeobot. 1: 3-17.

Gaillard M.-J., Birks H. J. B., Emanuelsson U., Karlsson S., Lagerls L. \& Olausson D., 1994, Application of modern pollen/land-use relationships to the interpre- 
tation of pollen diagrams-reconstructions of land-use history in south Sweden, 3000-0 BP, Review of Palaeobotany and Palynology 82 (1/2): 47-73.

Galon R., 1958, Z problematyki wydm śródlądowych w Polsce [Sur les dunes continentales en Pologne], [in:] R. Galon (ed.), Wydmy śródlądowe Polski, część pierwsza [Les dunes continentales en Pologne, premier partie], PWN, Warszawa: 13-31.

Hammer $\breve{R}$., Harper, D.A.T. \& Ryan P. D., 2001, PAST: Paleontological Statistics Software Package for Education and Data Analysis, Palaeontologia Electronica 4 (1), http:// palaeo-electronica.org/2001 1/past/issue 1 01.htm.

Hrynowiecka-Czmielewska A., Filbrant-Czaja A. \& Nienartowicz A., 2007, Modern pollen - vegetation relationships in the Tuchola Forest, Ecological Questions 8: $47-70$.

Jackson S. T., 1990, Pollen source area and representation in small lakes of the Northeastern United States, Review Palaeobotany and Palynology 63: 53-76.

Jackson S. T., 1991, Pollen representation of vegetational patterns along an elevation gradient, J. Veg. Sci. 2: 613-624.

Janssen C. R., 1996, Recent pollen spectra from the deciduous and coniferous-deciduous forests of north-western Minnesota: a study of pollen dispersal, Ecology 47: 804-825.

Janssen J. G. M., 1975, a simple clustering procedure for preliminary classification of very large sets of phytosociological relevés, Vegetatio 30: 67-71.

Jarzębski M., Nienartowicz A., Deptuła M., Bubnicki J. \& Domin D. J., 2010, Past, current and potential resources of carbon and above-ground plant biomass In the landscape with heaths in Tuchola Forest, Ecological Questions 13: 9-27.

Kavadze E.V., 1996, Recent pollen spectra of the mountain forest of the Lagodekhi Reservation (East Georgia), Acta Palaeobotanica 36: 121-147.

Kovach W. L., 1985-1999, MVSP PLUS version 3.1, Pentraeth, UK.

Kujawa-Pawlaczyk J., 2004, Suche wrzosowiska (CallunoGenistion, Pohlio-Callunion, Calluno-Arctostaphylion) [Dry heathlands (Calluno-Genistion, Pohlio-Callunion, Calluno-Arctostaphylion)], [in:] J. Herbich (ed.), Murawy, łąki, ziołorośla, wrzosowiska, zarośla, Poradniki ochrony siedlisk i gatunków Natura 2000 - podręcznik metodyczny, tom 3 [Grasslands, meadows, tall herb vegetation, thickets, Guides to conservation of habitats and species of Natura 2000 - The methodical guidebook, vol. 3], Ministerstwo Środowiska, Warszawa: 32-48.

Maarel van der E., 1979, Transformation of cover-abundance values in phytosociology and its effects on community similarity, Vegetatio 39: 97-114.
Makohonienko M, Gaillard M.-J. \& Tobolski K., 1993, Modern pollen/land-use relationships in ancient cultural landscape of North-Western Poland, with an emphasis on mowing, grazing and crop cultivation, [in:] B. Frezel (ed.), Quantification of land pollen/vegetation/landscape relationships as an aid to the interpretation of fossil pollen data, Paläoklimforschung 27, Special Issue: ESF Project "European Palaeoclimate and Man” 18, Gustav Fischer Verlag: 85-101.

Matuszkiewicz W., 2007, Przewodnik do oznaczania zbiorowisk roślinnych Polski [Guide for identification of plant communities in Poland], Vademecum Geobotanicum, WN PWN, Warszawa.

Mrózek W., 1958, Wydmy Kotliny Toruńsko-Bydgoskiej [Dunes of the Toruń Basin], [in:] R. Galon (ed.), Wydmy Śródlądowe Polski, część druga [Les dunes continentales en Pologne, deux partie], PWN, Warszawa: $7-59$.

Mulder Ch., 1997, Actuopalynology and Mediterranean vegetation patterns along the littoral of southern Latium (Italy), Actuopalynology and Mediterranean vegetation patterns 58: 285-291.

Nalepka D., \& Walanus A., 2003, Data processing in pollen analysis, Acta Palaeobotanica 43 (1): 125-134.

Nienartowicz A., Kunz M., Adamska E., Boińska U., Deptuła M., Gugnacka-Fiedor W., Kamiński D. \& Rutkowski L., 2010, Relief and changes in the vegetation cover and the flora of the Zadroże Dune near the city of Torun: comparison of the conditions in 1948 and 2009, Ecological Questions 12: 17-49.

Noryśkiewicz A. M., 1997, Analiza palinologiczna ściółki leśnej rezerwatu "Cisy Staropolskie" im. Leona Wyczółkowskiego we Wierzchlesie [Palynological analysis of forest litter in the Leon Wyczółkowski Nature Reserve „The Old Polish Yews” in Wierzchlas], [in:] E. Krasicka-Korczyńska (ed.), Ochrona gatunkowa na obszarach chronionych [Species protection in protected areas], Towarzystwo Miłośników Borów Tucholskich, Tuchola: 55-61.

Orlóci L., 1978, Multivariate Analysis of Vegetation Research, Dr. W. Junk Publishers, The Hague, Boston.

Parsons R. W., Prentice I. C. \& Saarnisto M., 1980, Statistical studies on pollen representation in Finnish lake sediments in relation to forest inventory data, Annales Botanici Fennici 17: 379-393.

Pidek I. A., Svitavská-Svobodova H., van der Knaap W. O., Noryśkiewicz A. M., Filbrandt-Czaja A., Noryśkiewicz B., Latałowa M, Zimny M., Święta-Musznicka J., Bozilova E., Tonkov S., Filipova-Marinova M., Poska A., Giesecke T. \& Gikov A., 2010, Variation in annual pollen accumulation rates of Fagus along a N-S transect in Europe based on pollen traps, Veg. Hist. Archaeobot. 19: 259-270. 
Prentice I. C., Berglund B. E. \& Olsson T., 1987, Quantitative forest-composition sensing characteristics of pollen samples from Swedish lakes, Boreas 16: 43-54.

Stockmarr J., 1971, Tablets with spores used in absolute pollen analysis, Pollen et Spores 13: 615-621.

Sugita S., 1994, Pollen representation of vegetation in quaternary sediments: theory and method in patchy vegetation, Journal of Ecology 82: 881-897.

Sugita S., Gaillard M. J. \& Broström A., 1999, Landscape openness and pollen records: a simulation approach, The Holocene 9: 409-421.

Tauber H., 1967, Investigations of the mode of pollen transfer in forested areas, Review of Palaeobotany and Palynology 3: 277-286.
Tauber H., 1974, a statistic non-overload pollen collector, New Phytol. 73: 359-369.

Trampler T., Kliczkowska A., Dmyterko E. \& Sierpińska E., 1990, Regionalizacja przyrodniczo-leśna na podstawach ekologiczno-fizjograficznych [Nature and forest regionalization on the ecological and physiographic basis], Państwowe Wydawnictwa Rolnicze i Leśne, Warszawa.

Walanus A. \& Nalepka D., 1999, POLPAL Program for counting pollen grains, diagrams plotting and numerical analysis, Acta Palaeobotanica (Supplemantum) 2: 659-661. 